\title{
A feedback-loop between telomerase activity and stemness factors regulates PDAC stem cells
}

Karolin Walter ${ }^{1}$, Eva Rodriguez-Aznar ${ }^{1}$, Monica S. Ventura Ferreira ${ }^{2}$, Pierre-Olivier Frappart ${ }^{1}$, Tabea Dittrich ${ }^{1}$, Kanishka Tiwary ${ }^{1}$, Sabine Meessen ${ }^{3}$, Laura Lerma ${ }^{4}$, Nora Daiss ${ }^{1}$, Lucas-Alexander Schulte ${ }^{1}$, Frank Arnold ${ }^{1}$,

Valentyn Usachov ${ }^{1}$, Ninel Azoitei ${ }^{1}$, Mert Erkan ${ }^{4,5}$, Andre Lechel ${ }^{1}$, Tim H. Brümmendorf ${ }^{2}$, Thomas Seufferlein ${ }^{1}$, Alexander Kleger ${ }^{1}$, Enrique Tabarés ${ }^{6}$, Cagatay Günes ${ }^{3}$, Fabian Beier ${ }^{2}$, Bruno Sainz, Jr. 7,8,9, Patrick C. Hermann ${ }^{1}$

${ }^{1}$ Department of Internal Medicine I, University Medical Centre Ulm, 89081 Ulm, Germany

${ }^{2}$ Department of Hematology, Oncology, Hemostaseology and Stem Cell Transplantation, University Hospital of the RWTH Aachen, 52062 Aachen, Germany

${ }^{3}$ Department of Urology, Ulm University, 89081 Ulm, Germany

${ }^{4}$ Department of Surgery, Koç University School of Medicine, 34450 Istanbul, Turkey

${ }^{5}$ Research Center for Translational Medicine, Koç University, 34450 Istanbul, Turkey

${ }^{6}$ Department of Preventive Medicine, Public Health and Microbiology, Universidad Autónoma de Madrid (UAM), 28049 Madrid, Spain

${ }^{7}$ Department of Biochemistry, Universidad Autónoma de Madrid (UAM), 28049 Madrid, Spain

${ }^{8}$ Department of Cancer Biology, Instituto de Investigaciones Biomédicas “Alberto Sols” (IIBM), CSIC-UAM, 28049 Madrid, Spain

${ }^{9}$ Chronic Diseases and Cancer, Area 3 - Instituto Ramón y Cajal de Investigación Sanitaria (IRYCIS), 28049 Madrid, Spain

\section{Correspondence:}

PD Dr. Dr. Patrick C. Hermann

Department of Internal Medicine I, University Medical Centre Ulm

Albert-Einstein-Allee 23

89081 Ulm, Germany

Phone: +49-731-500-44736

Email: patrick.hermann@uni-ulm.de

Running title: Role of telomerase in PDAC stem cells 


\section{Abstract}

To date, it is still unclear how cancer stem cells (CSCs) regulate their stemness properties, and to what extent they share common features with normal stem cells. Telomerase regulation is a key factor in stem cell maintenance. In this study, we investigate how telomerase regulation affects cancer stem cell biology in pancreatic ductal adenocarcinoma (PDAC), and delineate the mechanisms by which telomerase activity and CSC properties are linked. Using primary patientderived pancreatic cancer cells, we show that CSCs have higher telomerase activity and longer telomeres than bulk tumor cells. Inhibition of telomerase activity, using genetic TERT-knockdown or pharmacological inhibitor (BIBR1532) resulted in CSC marker depletion in vitro, and reduced tumorigenicity in vivo. Furthermore, we identify a positive feedback loop between stemness factors (KLF4, SOX2, OCT3/4, NANOG) and telomerase, which is essential for the self-renewal of pancreatic CSCs. Disruption the balance between telomerase activity and stemness factors, eliminates CSCs via induction of DNA damage and apoptosis, opening future perspectives to avoid CSC driven therapy resistance and tumor relapse in PDAC patients.

Key words: cancer stem cells/ pancreatic ductal adenocarcinoma/ self-renewal/ stemness/ telomerase activity 


\section{Introduction}

Pancreatic ductal adenocarcinoma (PDAC) is the most frequent and the most lethal form of pancreatic cancer, and is expected to be the second most frequent cause of cancer-related death by 2030 (Rahib et al, 2014). Diagnosis of PDAC is frequently delayed due to the absence of early symptoms, pronounced resistance to therapy and early metastatic spread. As a consequence, less than $20 \%$ of patients diagnosed with PDAC are eligible for resection (Siegel et al, 2015), the only curative treatment option. Despite growing knowledge about PDAC tumor biology, advances in treatment are still scarce, with FOLFIRINOX and gemcitabine + nab-paclitaxel currently representing the most promising combination chemotherapies (Conroy et al, 2011; Von Hoff et al, 2013). The complexity and heterogeneity of pancreatic cancer remains only partially deciphered, and strategies for developing novel and more effective treatments are urgently needed.

Cancer stem cells (CSCs) have been implicated in a wide variety of tumors. We and others have previously demonstrated their outstanding importance in pancreatic cancer perpetuation, metastasis, and therapy resistance.(Hermann et al, 2007; Li et al, 2007) While self-renewal is a defining characteristic of CSCs, (Clarke et al, 2006) the precise mechanism how CSCs maintain their stemness state remains unclear. Therefore, understanding CSCs at the molecular level might reveal targetable principles that could potentially be exploited therapeutically. Since telomerase activity and telomere regulation have been proposed to play an essential role in tumor cell maintenance and are considered "hallmarks of cancer" by Hanahan and Weinberg, telomerase activity might present such a potential target.

Vertebrate telomeres consist of repetitive TTAGGG DNA sequences. The telomerase complex consists of a catalytic subunit (telomerase reverse transcriptase, TERT) and a telomerase RNA component (TERC). TERC serves as a template for the addition of DNA tandem repeats catalyzed by TERT, which is the rate-limiting factor for telomerase activity. (Nakamura, 1997) Further factors such as the shelterin proteins TERF1 and TERF2 represent important factors for telomerase regulation. (DeLange, 2005) Telomeres protect the ends of the chromosomes from endto-end fusion and exonucleolytic degradation, preventing genome instability. Importantly, in most human cells telomeric DNA is shortened with each cell division, leading to undetectable telomerase activity in the vast majority of somatic tissues (Wright et al, 1996) and accumulation of critically short telomeres, subsequently limiting cellular replicative capacity and ultimately resulting in replicative senescence. (Collado et al, 2007)

Telomere length stabilization by the reactivation of telomerase or (much less frequently) through alternative mechanisms of telomere lengthening (ALT) results in elevated telomerase activity in $85-90 \%$ of human tumors, highlighting this as the prime mechanism to maintain telomere functionality in cancer. (Shay \& Wright, 2019) 
Several studies have examined the effects of telomerase inhibition on tumor growth. In cancer cell lines, the potent non-nucleosidic telomerase inhibitor BIBR1532 induces telomere shortening, proliferation arrest and senescence (Damm et al, 2001). Currently the effects of the clinical grade telomerase inhibitor GRN163L (Imetelstat) are being tested in several tumors, such as breast and lung cancer, myeloma. However, the effects of telomerase inhibition on CSCs have yet to be elucidated.

In the present study we investigate the role of telomere regulation and telomerase inhibition in the maintenance of pancreatic CSCs. We demonstrate that CSCs isolated from patient-derived xenografts (PDXs) present higher telomerase activity, resulting in significantly longer telomeres compared to bulk tumor cells. Intriguingly, the lengthening of telomeres is inextricably linked to increased expression of the pluripotency / stemness factors OCT3/4, SOX2, NANOG and KLF4, and is jointly regulated in a previously undescribed positive feedback loop which is necessary for these cells to escape senescence, and regulates their stemness properties. Furthermore, pharmacological inhibition of telomerase using BIBR1532 as well as genetic knock-down of TERT greatly decreased the CSC frequency of patient-derived PDAC cells in vitro and in vivo by CSC-specific induction of DNA damage and apoptosis. 


\section{Results}

\section{Telomerase activity and telomere length in pancreatic CSCs vs bulk tumor cells.}

In a first screen we tested several primary PDAC cell lines for their average telomere length. Indeed, we observed that all tested samples showed very short telomeres $(<5$ th age percentile) $($ Fig. 1A). In order to evaluate telomerase activity specifically in CSCs, we compared the expression levels of TERT and TERF 1 in CSCs versus bulk tumor cells in three human PDX-derived primary PDAC cell lines (Panc215, Panc185, Panc354). Using well-established CSC enrichment methods (i.e. CD133 ,or Aldefluor fluorescence activated cell sorting (FACS), and sphere culture (Fig. 1B)), we found increased expression of TERT and/or TERF1 in CD133+ cells (Fig. 1C), Aldefluor+ cells (Fig. 1D) and in sphere cultures (Fig. 1E) compared to the negative population or adherent cells, respectively. In immunofluorescence staining, significantly more TERT-expressing cells were detectable in the CD133+ CSC subpopulation as compared to CD133- cells (Fig. 1F). Next, we measured telomerase activity as the functionally relevant readout. Using gel-based as well as PCR-based TRAP (telomeric repeat amplification protocol) assays, significantly higher telomerase activity was detected in the CSC fraction as compared to the respective control cells (Fig. 1G-I). Since the key function of telomerase is telomere elongation, we next performed Q-FISH-analysis to determine telomere length in CD133+ (Fig. 1J) or ALDH+ (Supp Fig. S1A) CSCs and in the respective marker-negative control populations (Panc215: CD133+ 66.2 a.u. vs CD133- 31.7 a.u.; Panc185: CD133+ 40.8 a.u. vs CD13331.5 a.u.; Panc354: CD133+ 41.6 a.u. vs CD133- 35.4 a.u.; and Panc286: ALDH+ 11.2556 a.u. vs ALDH- 9.1304 a.u.). Indeed, we observed significantly longer telomeres in the investigated CSC populations. These results confirm increased telomerase expression and activity in pancreatic CSCs as compared to non-CSCs.

\section{A positive feedback loop between stemness factors and telomerase maintains CSC phenotype}

Since we observed a significant increase in the regulation and activity of telomerase in CSCs, we next investigated whether increased telomerase activity is associated with the expression of genes and markers of stemness and pluripotency. We observed increased expression of OCT3/4, NANOG, and LGR5 in the CSC population (Fig. 2A) as compared to the CD133- control population. Similarly, expression levels of these genes and of SOX2 were significantly elevated in Aldefluor-positive cells (Fig. 2B) compared to the negative cells, demonstrating increased expression of most stemnessassociated genes in CSCs with some variation depending on the utilized identification strategy. To overcome CSC marker-dependent variation, we next infected Panc354 primary cell cultures with a Nanog-YNL reporter system. (Takai A., 2015) YNL+ cells showed increased expression of NANOG, but also of TERT and other stemness-associated genes (Fig. 2C). Furthermore, YNL+ cells showed 
significantly higher telomerase activity (Fig. 2D). While the amount of CD133+ cells was unchanged (Fig. 2E), sphere forming capacity was significantly increased (Fig. 2F) in the YNL+ cells.

To confirm higher telomerase activity in CSCs, we used a recombinant pseudorabies virus (PRV) that we previously designed and demonstrated to replicate only in cells with high telomerase activity (PRV-TER). (Lerma et al, 2016) Adherent cultures of human pancreatic ductal epithelial (HPDE) cells and Panc185 cells were infected with two parental control viruses (PRV-NIA3 and vBecker2) and with PRV-TER. While both parental viruses efficiently replicated in HPDE and Panc 185 cultures, PRV-TER was unable to produce de novo virions in either culture (Fig. 2G). In contrast, when CSCenriched sphere cultures were infected, all three viruses efficiently replicated and produced de novo virions, confirming that CSC-enriched sphere cultures express significantly higher telomerase activity, allowing for PRV-TER to efficiently replicate in these cultures.

Finally, we explored the mechanism(s) underlying increased telomerase regulation in CSCs. Direct promoter activation can lead to increased TERT activity in tumor cells, and two common promoter mutations (-124 and -146 bp upstream of the TERT start site) have been associated with increased telomerase activity. (Vinagre et al, 2013) These mutations are frequent in some cancers, but rarely occur in gastrointestinal cancers. Indeed, promoter sequencing of all tested PDAC cells revealed wildtype sequences (6/6 primary cell lines, 5/5 established cell lines, Fig. $2 \mathbf{H}$ and Supp Fig. S1B).

We next generated PiggyBac expression vector constructs that specifically express $S O X 2$, OCT3/4, KLF4 or NANOG to determine how these stemness factors modulate TERT expression. Supporting this approach, Hsieh et al. showed that PARP1 recruits KLF4 to activate telomerase expression in embryonic stem cells. (Hsieh et al,2017) Interestingly, the expression of OCT3/4 and SOX2 significantly upregulated TERT expression and a similar trend was observed for NANOG and KLF4 (Fig. 2I). Furthermore, overexpression of any of these factors resulted in concomitant upregulation of the other factors as well, indicating an intricate cross-regulation of these individual stemness factors. Taken together, these data show that telomerase activity is increased in CSCs and is regulated by a feedback loop between TERT and key pluripotency-associated factors.

\section{Telomerase inhibition causes DNA damage and apoptosis in CSCs.}

Based on the observed link between telomerase activity / telomere length and a CSC phenotype, we next evaluated the effects of telomerase inhibition on the CSC population. For this purpose, we treated primary pancreatic cancer cells with the small molecule telomerase inhibitor BIBR1532 and selected $80 \mu \mathrm{M}$ as an effective sub-IC 50 concentration (Supp Fig. S1C). BIBR1523 treatment was performed for $3 \mathrm{~d}$ or $7 \mathrm{~d}$, respectively, and after $7 \mathrm{~d}$ of treatment telomerase activity was effectively decreased (Fig. 3A). Functionally, treatment with BIBR1523 significantly reduced telomere length (Fig. 3B) 
already after $3 \mathrm{~d}$. Interestingly, the treatment effect was potentiated with longer treatment in the CD133+ CSC population. However, in the CD133- bulk tumor cell population telomere length increased again after $7 \mathrm{~d}$ of treatment. This is most likely due to early elimination of CD133- cells with critically short telomeres and the inability of CD133- cells to upregulated telomerase, leading to a relative enrichment in CD133- cells with longer telomeres. Based on these results, treatment with BIBR1523 was performed for $7 \mathrm{~d}$ in all further experiments.

To determine the biological consequence of telomerase inhibition, we quantified DNA damage by $\gamma-\mathrm{H} 2 \mathrm{AX}$ staining, as telomerase inhibition can induce DNA damage, cell cycle arrest and apoptosis in other systems. (Celeghin et al, 2016) Treatment with BIBR1532 increased $\gamma-\mathrm{H} 2 \mathrm{AX}$ foci generation much more strongly in CD133+ CSCs than in bulk tumor cells, indicating a preferential induction of DNA damage after telomerase inhibition in CSCs (Fig. 3C). Accumulation of DNA double strand breaks (DSBs) and the activation of DNA damage response are considered contributing factors to cellular senescence;(Collado et al., 2007) Therefore, we next measured senescence using flow cytometry to detect $B$-galactosidase after BIBR1532 treatment and observed increased senescence in both CD133- and CD133+ cells, excluding preferential induction senescence in CSCs as response to BIBR1532 treatment (Fig. 3D).

Since DSBs can also induce apoptosis, we next measured early apoptotic cells by AnnexinV staining and observed significantly more apoptotic cells in the CD133+ CSC population upon BIBR1532 treatment, while CD133- cells were unaffected (Fig. 3E). Furthermore, we performed Caspase 3/7 staining to determine whether BIBR1532-induced DNA damage leads to the recruitment of apoptosis effector kinases. In line with the AnnexinV staining, BIBR1532 treatment resulted in increased Caspase 3/7 staining in the CD133+ CSC population, while no differences were observed in CD133- cells (Fig. 3F).

\section{CSCs are depleted upon telomerase inhibition.}

Since BIBR1532 treatment appeared to have CSC-specific effects, we next evaluated the effect of telomerase inhibition on CSC properties. As expected, BIBR1532 treatment strongly reduced the expression of TERT, along with established stemness / pluripotency-associated genes (Fig. 4A), and significantly decreased the percentage of CSCs as measured by expression of CD133 or Aldefluor (Fig. 4B, Supp Fig. S1D, S1E) or sphere formation capacity (Fig. 4C). Most importantly, BIBR1532 treatment strongly reduced the number of tumor-initiating cells in (extreme limiting dilution (ELDA) tumorigenicity assays in nude mice. But also the tumor growth of the 100.000 injected cells after BIBR1532 treatment was significantly reduced compared to the tumor growth of solvent treated cells (Fig. 4D). These data clearly indicate that telomerase inhibition by BIBR1532 strongly affects the functional capacity of the CSC population. 
We have previously shown that chemotherapy enriches for CSCs, and have demonstrated the efficacy of combining stem cell inhibitors, stroma-depleting agents and chemotherapy to significantly improve survival of mice in vivo.(among others:(Lonardo et al, 2011; Mueller et al, 2009)) In organoid cultures generated from primary pancreatic cancer cells we observed a significant decrease in luminescence (i.e. viability) upon treatment with increasing doses of gemcitabine. However, gemcitabine did not abrogate organoid formation, which was only achieved using BIBR1532 treatment (Fig. 4E). Since the induction of DNA damage by telomerase inhibition did not entirely abrogate CSC function (see Fig. 3), we used a combination of chemotherapy, BIBR1532 and the PARP inhibitor Olaparib to target CSCs more successfully. Indeed, evaluating sphere formation as surrogate marker for CSC activity, the combination therapy had significantly stronger effects than chemotherapy alone (Fig. 4F).

\section{Telomerase inhibition as novel treatment strategy for pancreatic cancer.}

In order to ensure that the effects of BIBR1532 are specific to telomerase inhibition, we proceeded to corroborate our main findings with shRNA-mediated TERT-knockdown (TERT-KD). As expected, genetic knockdown of TERT using two different shRNAs suppressed the expression of TERT (Fig. 5A), and strongly suppressed telomerase activity (Fig. 5B); due to its stronger effects, shRNA 340160 was used for all subsequent experiments. TERT-KD suppressed the expression of stemness-associated genes (Fig. 5C). The reduction in stemness translated into depletion of CD133+ CSCs (Fig. 5D) and reduced sphere formation activity (Fig. 5E). Similar to the effects of BIBR1532 (Fig. 3F\&G), the TERT-KD significantly increased apoptosis in CD133+ cells but not in CD133cells (Fig. 5F). Most importantly, the TERT-KD strongly reduced TIC frequency in ELDA xenografting assays (Fig. 5G, Suppl. Fig 1F). These results closely resemble those achieved with BIBR1532, confirming that the effects observed above are indeed telomerase-specific.

In order to model the effects of telomerase inhibition as an applied treatment in vivo, we performed subcutaneous xenografting of Panc215 cells carrying an inducible shTERT cassette. Induction of the TERT-KD before injection with doxycycline resulted in significantly smaller tumors compared to scrambled control (Fig. 5H). In a preclinical trial we xenografted inducible TERT-KD Panc215 cells and induced the knockdown 28 days after implantation, when tumors were firmly established. While dox-treated scrambled cells showed continuous tumor growth leading to sacrifice of the mice, we observed disease stabilization after TERT-KD (Fig. 5I). The residual tumors showed downregulation of $h T E R T$ as well as decreased telomerase activity (Fig. 5J). Histological analysis of the explanted tumors showed no gross differences between the two treatment groups with regard to tumor morphology, cellularity or stroma composition (Fig. 5K). 
237 Altogether, these data indicate that TERT/telomerase inhibition depletes CSCs, resulting in disease 238 stabilization in PDAC. 


\section{Discussion}

We and others have previously identified CSCs in pancreatic cancer, and have demonstrated that they play a key role in the propagation, chemoresistance and metastasis (Hermann et al., 2007; Li et al., 2007). PDAC remains a disease that is terribly difficult to treat. Thus, eliminating CSCs as a continuous source of tumor growth, resistance and relapse is of utmost importance for improved clinical outcome. While functional differences in CSCs are continuously being discovered, the underlying mechanisms for their acquisition of stemness features are much less clear.

CSCs have been isolated based on different markers and cellular features. We have previously established CD133 expression and functional enrichment via sphere formation as reliable CSC markers in PDAC, (Hermann et al., 2007) and together with Aldefluor activity these are most widely used. In addition to using these markers, we evaluated the expression levels of pluripotencyassociated genes such as NANOG, OCT3/4, KLF4, SOX2 and others to overcome the limitations of the markers mentioned above, and additionally used a NANOG reporter system to further demonstrate stemness in the isolated CSCs.

Unlimited proliferation requiring telomere maintenance is a "hallmark of cancer" as indicated by Hanahan \& Weinberg, and selective telomere maintenance or even elongation via telomerase activity is an essential feature of stem cells. However, available data on telomerase activity and its regulation in CSCs is scarce.

Increased telomere length has been reported in several tumor entities, such as prostate cancer and glioblastoma. (Beier et al, 2011; Marian et al, 2010) In contrast to these studies and our data, Joseph et al. surprisingly observed no such differences in breast and pancreatic cancer cells. (Joseph et al, 2010) This might be explained by the use of only one established cell line versus the panel of primary cells used in our study, as well as by inter-tumoral heterogeneity. Interestingly, Joseph et al. did observe reduced tumor engraftment in nude mice under treatment with the telomerase inhibitor Imetelstat, indicating an effect on a CSC population. While a telomerase-independent mechanism of regulation was suggested by Joseph et al., and the use of high doses of BIBR1532 has also been shown to induce cytotoxicity irrespective of telomerase activity, (El-Daly et al, 2005) our data clearly demonstrate the dependency of CSCs on intact telomere regulation. Specifically, we demonstrate that pharmacological inhibition or shRNA-mediated TERT-KD result in CSC depletion as evidenced by reduced expression of stemness genes and surface markers, and abrogation of sphere formation and tumorigenicity in vivo. While telomere length can be maintained by alternative lengthening of telomere (ALT) mechanisms in 10-15\% of the tumors, the activation of ALT pathways is rare in cancer and most cancers maintain telomeres through regulation of telomerase activity. (Shay \& Wright, 2019) Furthermore, the telomere staining pattern observed in our cells shows no indication for an important role of ALT. 
Direct promoter activation may also lead to increased TERT activity in tumor cells, and two common promoter mutations (-124 and -146 bp upstream of the TERT start site) are typically associated with increased telomerase activity. While these mutations are frequent in other cancers, they rarely occur in GI-tumors, (Vinagre et al., 2013) which is well in line with our own observation that all PDAC cells we tested showed wildtype promoter sequences.

Using BIBR1532 as a small molecule telomerase inhibitor, we detected a dose-dependent proliferation arrest, which is in line with reduced cell proliferation observed in ESCs. Critical shortening of telomeres induces DNA damage response pathways, cell-cycle arrest and finally cell death. These effects are triggered by ATM and/or ATR-dependent signaling via checkpoint activation, resulting in the marking of uncapped chromosomal ends by formation of $\mathrm{gH} 2 \mathrm{AX}$ foci and telomere dysfunction-induced foci (TIFs).(Sexton et al, 2014) Thus, the cellular response to dysfunctional telomeres is regulated through the same factors that control DNA damage response (Takai et al, 2003) and activate p53 and its downstream targets.(Brassat et al, 2011) We have previously demonstrated that CD133+ CSCs exhibit enhanced DNA damage repair and are particularly sensitive to inhibition of ATR-mediated DNA damage response.(Gallmeier et al, 2011)

Indeed, we observed that BIBR1532 treatment resulted in $\mathrm{gH} 2 \mathrm{AX}$ foci formation and senescence in CD133+ cells. These effects were accompanied by preferential induction of apoptosis in the CSC population, emphasizing the dependency of CSCs on functional telomerase and stabilized telomeres. CSCs also appear to be more susceptible to telomerase inhibition-induced DNA damage and ultimately to the induction of apoptosis, which is especially interesting since other stem cells (ESCs and iPSCs) are particularly sensitive to DNA damage-induced apoptosis. (Liu et al, 2016) is crucial to improve therapy efficacy and outcome for PDAC patients. We have repeatedly shown that the identification of stem cell features in CSCs, such as the activity of embryonic signaling pathways can result in novel treatment strategies to eliminate pancreatic CSCs, resulting in long-term survival of mice harboring PDAC. (Lonardo et al., 2011; Mueller et al., 2009) Here we demonstrate a new approach to this goal and show that pancreatic CSCs are highly dependent on telomerase activity and telomere elongation, and that loss of telomerase activity by genetic knockdown or small molecule inhibitors eliminates patient-derived pancreatic CSCs. For this study we used a series of primary cell lines generated from patient-derived xenografts. While this inevitably leads to more heterogeneity when it comes to results, it is important to note that the underlying mechanisms as well as the effects of telomerase inhibition, both by small molecules and TERT-KD, are reproducible in all utilized cell lines, strongly supporting the significance of the results.

The prediction of drug response in vitro on 2D monolayer cultures is traditionally very difficult. 3D pancreatic organoid cultures better recapitulate the situation in a tumor and are superior 
to 2D cultures when it comes to drug testing.(Griffith \& Swartz, 2006) Therefore, we generated organoid cultures from patient-derived xenografts for drug testing and detected a dose-dependent response of organoid viability to both BIBR1532 and gemcitabine treatment. To demonstrate the efficacy of a drug regimen against CSCs, however, tumor formation (or lack thereof) in vivo remains the gold standard. Therefore, we used an inducible TERT-KD construct, allowing for treatment to commence after tumor formation and growth, replicating the therapeutic scenario in a clinical setting without the confounding off-target effects of small molecule inhibitors. Indeed, we observed significantly smaller tumors whenever telomerase function was lost, indicating a crucial role for TERT in tumor propagation.

TERT-KD depletes CSCs, highlighting the dependency of CSCs on intact telomerase regulation. In this respect CSCs seem resemble human ESCs, where TERT has been shown to be an essential mediator of pluripotency, cell cycle progression and differentiation.(Liu, 2017; Yang et al, 2008) furthermore, we observed that TERT-KD critically downregulates the core pluripotency factors OCT4, KLF4, SOX2 and NANOG, and KLF4 is known to bind and activate the TERT promoter in human and mouse stem cells. (Hoffmeyer et al, 2012; Wong et al, 2010) However, many of the transcription factors involved in the regulation of self-renewal and pluripotency in human ESCs are tightly intertwined; KLF4 binds and upregulates the NANOG promoter, which in turn is also regulated through cooperation of SOX2 and OCT4 with KLF4.(Chan et al, 2009; Nakatake et al, 2006) Furthermore, silencing of OCT3/4 correlates with downregulation of pluripotency factors and TERT, with simultaneous activation of p53 and upregulation of its target genes p21 and PUMA.(Zhang et al, 2014) The subsequent p53-dependent induction of cell cycle arrest, senescence, apoptosis and the suppression of pluripotency and self-renewal in ESCs as a result of DNA damage has also been demonstrated. (Chin et al, 1999) Thus, a tightly coordinated regulation of stemnessassociated transcription factors and pathways is essential for the preservation of "stemness".

We therefore hypothesized that TERT expression is regulated by the same transcription factor signature; Using a NANOG reporter system we demonstrate that high NANOG expression correlates directly with TERT expression and vice versa, conferring increased CD133 expression and sphere formation ability to cancer cells. Using expression vectors for OCT3/4, SOX2, KLF4 and NANOG, we demonstrate that while all four pluripotency factors seem to increase TERT expression and thus impact the regulation of telomerase activity, SOX2 and OCT3/4 do so most strongly. In summary, these data show a previously undiscovered positive feed-back loop between pluripotency transcription factors and TERT regulation in pancreatic CSCs.

TERT is furthermore regulated by epigenetic changes, such as chromatin loop structures in cells with long telomeres (Kim et al, 2016) or through CpG islet methylation of the TERT promoter. However, epigenetic changes of TERT by methylation remain a controversial topic: Bechter et al. 
344 demonstrated that in patients with B-CLL, TERT promoter hypomethylation was associated with an 345 increased telomerase activity, (Bechter et al, 2002) whereas others showed that TERT 346 hypermethylation increases TERT mRNA expression and telomerase activity in a variety of cancers 347 (e.g. bladder, brain, colon, heart and kidney) (Guilleret et al, 2002). We found no differences in TERT 348 promoter methylation using previously published global methylation data, suggesting that 349 methylation is likely not a controlling factor in pancreatic CSCs.

350 In summary, the results presented in this study demonstrate that telomerase regulation is critical for 351 the maintenance of stemness in CSCs. This may significantly promote our understanding of PDAC tumor biology and may eventually result in improved treatment for pancreatic cancer patients. 


\section{Materials and Methods}

\section{Mice, transplantation and treatment}

Female 6-8 week-old athymic Nude-Foxn $1^{\text {nu }}$ mice were purchased from Envigo (France). For subcutaneous xenografting, single cells were resuspended in $40 \mu 1$ 1:1 media:Matrigel (Invitrogen). CSC frequencies and statistical significance of the comparison were determined from extreme limiting dilution assay results using ELDA software.(Hu \& Smyth, 2009) For in vivo treatment, animals received biweekly intraperitoneal (i.p.) injections of doxycycline (1 mg/ml). Tumor size was calculated using the formula (length $\mathrm{x}$ width $\left.{ }^{2}\right) / 2$. Mice were housed and maintained in laminar flow cabinets in specific pathogen-free conditions. All animal work and care were carried out following strict guidelines following German or Spanish legal regulations to ensure careful, and ethic handling of mice. The experimental protocol for animal studies was reviewed and approved by the respective government review board and institutional animal care.

\section{Primary pancreatic cancer cells and other cell lines}

Primary human pancreatic cancer cell lines were generated from established PDX obtained under MTAs (Reference no. I409181220BSMH and I405271505PHMH) from the CNIO, Madrid, Spain, and maintained in culture as described previously. (Mueller et al., 2009) U-2OS and HPDE cells have been previously described.(Lerma et al., 2016) Hek293T cells were cultured in DMEM supplemented with $10 \%$ FBS, $1 \%$ Pen/Strep and $1 \%$ Glutamine, in standard conditions $\left(37^{\circ} \mathrm{C}, 5 \%\right.$ $\mathrm{CO}_{2}$ ). BIBR1532 (Selleckchem) was used at $80 \mu \mathrm{M}$ (3 or 7 days treatment, changing media and BIBR1532 every other day), Gemcitabine (Merck) was used at 5 $\mu \mathrm{M}$ and Olaparib (Selleckchem) was used at $25 \mu \mathrm{M}$ (for $72 \mathrm{~h}$ ) for 3D cell culture unless stated otherwise.

\section{CSC enrichment}

$\underline{\text { CD133 and ALDH fluorescence activated cell sorting (FACS) }}$

For CSC enrichment primary pancreatic cancer cells were stained with CD133 (See Additional file 1 Table 1) or the Aldefluor ${ }^{\mathrm{TM}}$ Kit (STEMCELL ${ }^{\mathrm{TM}}$ Technologies) was performed. Cell sorting was performed using a FACSAria III (BD Bioscience).

\section{$\underline{\text { Sphere culture }}$}

Spheres were cultured as described previously. (Hermann et al., 2007) Briefly, cells were cultured in DMEM-F12 supplemented with B-27 (ThermoFisher Scientific) and bFGF (Novoprotein). 10,000 cells per milliliter were seeded in ultra-low attachment plates (Corning). Spheres were defined as 3-dimensional multicellular structures of $\geq 40 \mu \mathrm{m}$. After 7 days, sphere formation was quantified either manually using a Leica stereomicroscope or using a CASY TT (OLS OMNI Life Science) with a $150 \mu \mathrm{m}$ capillary. Dead cells and debris were excluded from the quantification. 


\section{Pseudorabies virus infections}

The virulent Pseudorabies virus (PRV) strain PRV-NIA3 has been previously described. The parental PRV virus vBecker2 was generated by transfection of pBecker2 plasmid into Hela Tet-Off cells.(Lerma et al., 2016) PRV-TER, a recombinant PRV virus in which the endogenous viral IE180 promoter was substituted with the TERT human tumor promoter, has been detailed previously.(Lerma et al., 2016) For infection of HPDE and Panc185 cells in adherence, $5 \times 10^{5}$ cells were seeded in 6 multi-well plates. Twenty-four hours post seeding, cells were infected with PRV-NIA3, vBecker2 or PRV-TER at a multiplicity of infection (MOI) of 0.1 TCID $50 /$ cell. After an absorption period of $2 \mathrm{~h}$ at $37^{\circ} \mathrm{C}$, the cells were washed with PBS to remove unabsorbed virus, and incubated for $72 \mathrm{~h}$ at $37^{\circ} \mathrm{C}$. Virus yield was determined from total lysates of infected cells on U2OS cells as previously described.(Lerma et al., 2016) For infection of spheres, 7-day-old Panc185 $1^{\text {st }}$ generation spheres were trypsinized, cells were counted and cell suspensions of $5 \times 10^{5}$ cells were seeded in 6 multi-well plates and infected with PRV-NIA3, vBecker2 or PRV-TER at a multiplicity of infection (MOI) of $0.1 \mathrm{TCID} 50 /$ cell. After an absorption period of $2 \mathrm{~h}$ at $37^{\circ} \mathrm{C}$ under agitation, cultures were washed with PBS to remove unabsorbed virus, and incubated for $72 \mathrm{~h}$ at $37^{\circ} \mathrm{C}$. Virus yield was determined as detailed above.

\section{Telomerase activity}

For telomerase activity measurement, the TeloTAGGG telomerase PCR Elisa ${ }^{\text {PLus }}$ kit (Roche), the telomeric repeat amplification protocol (TRAP) or qRT-PCR based TRAP were performed. Primer sequences are provided in the Supplementary Information (See Supplementary file 1 Table 2).

\section{Organoid culture}

Tumor pieces of patient-derived xenografts (PDXs) were digested with Accutase ${ }^{\circ}$ solution (Merck) for $30 \mathrm{~min}$ at $37^{\circ} \mathrm{C}$. The cells were then filtered in EASY strainer $100 \mu \mathrm{M}$ (greiner bio-one) and cultured in Matrigel coated plates containing organoid culture medium as described in Dantes et al. 2017 with $5 \%$ growth factor reduced Matrigel (BD, 354230). Media of the organoid culture plates was refreshed every 3-4 days. Organoids were treated with BIBR1532 and gemcitabine at 12.5, 25.0 and $50 \mu \mathrm{M}$ for $72 \mathrm{~h}$. To determine the number of metabolically active and viable organoids, the CellTiter-Glo® 3D Cell Viability Assay (Promega, G9681) was performed following the manufacturer's instructions.

\section{Plasmids, infection and transfection}

For RNAi-mediated gene silencing, the pGIPZ and pTRIPZ lentiviral vectors developed by Dr. Greg Hannon (Cold Spring Harbor Laboratory) and Dr. Steve Elledge (Harvard Medical School) and the 
corresponding shRNA constructs (GIPZ TERT shRNAs, RHS4531-EG7015 and TRIPZ TERT shRNAs, RHS4740-EG7015) were purchased from Dharmacon. The NANOG reporter lentiviral vector backbone and the sequence of the construct have been previously described. (Hotta et al, 2009) NANOG, OCT3/4, SOX2 and KLF4 were cloned into the PB-EF1-MCS-IRES-RFP plasmid (SBI). Transposition was performed using Super PiggyBac transposase (SBI), transfection was performed with standard Lipofectamine 2000 (Invitrogen).

\section{Flow cytometry}

Antibodies used in this study are listed in the Supplementary file 1 (Table1). For ALDH detection the Aldefluor ${ }^{\mathrm{TM}}$ kit (STEMCELL ${ }^{\mathrm{TM}}$ Technologies) was used. Apoptosis was measured using Annexin V-APC (BD) and CellEvent ${ }^{\mathrm{TM}}$ Caspase-3/7 green detection reagent (invitrogen). For DNA content staining cells were stained with DAPI. Cellular senescence was measured using the SA- $\beta$-gal kit (BioCat). Samples were analyzed by flow cytometry using a LSR II (BD Bioscience), and data were analyzed with FlowJo V10 (Ashland, Oregon). FACSorting was performed using a FACSAria III (BD).

\section{Q-FISH}

Telomere Q-FISH was performed as previously described.(Hummel et al, 2015) Fluorescence intensity of the telomeres was quantified with Definiens software (Definiens, Munich, Germany).

\section{Immunofluorescence}

Primary pancreatic cancer cells were FACSorted, seeded on cover-slips in 6-well dishes, incubated at $37^{\circ} \mathrm{C}$ for $12 \mathrm{~h}$, washed with PBS and fixed with $2 \%$ PFA for $20 \mathrm{~min}$ at room temperature. Coverslips were washed 3 times with PBS and incubated at room temperature for $15 \mathrm{~min}$ in $0.7 \%$ triton in PBS. 1h blocking with 5\% milk powder in $0.1 \%$ TBS-T at room temperature was followed by incubation with the respective antibodies (1:500) in a humidified chamber at $4{ }^{\circ} \mathrm{C}$ overnight. Coverslips were then washed $3 \mathrm{x}$ with $0.1 \%$ TBS-T. Secondary antibodies $(1: 1,000)$ were incubated at room temperature in a humidified chamber for $2 \mathrm{~h}$, washed and mounted with ProLong ${ }^{\mathrm{TM}}$ Gold Antifade mounting reagent with DAPI (ThermoFisher). Images were captured using a Leica TCS SP8-HCS confocal microscope. Antibodies are listed in the Supplementary file (Table 1).

\section{RNA isolation and qRT-PCR}

Total RNA was prepared using RNeasy kits with on-column genomic DNA digestion following the manufacturer's instructions (Qiagen). First strand cDNA was prepared using QuantiTect Reverse Transcription kit (Qiagen). Reactions were performed with QuantiFastSybr Green PCR Kit (Qiagen) 
using a QuantStudio 3 machine (Applied Biosystems). Results were analyzed using the $2^{\text {-ddCt }}$ method and calculated as relative to $H P R T$ expression. Reactions were carried out from at least three independent experiments. Primer sequences are provided in the Supplementary file (Table 2).

\section{MTT assay}

1,000 cells were seeded in a 96 well plate and incubated for $24 \mathrm{~h}$ at $37^{\circ} \mathrm{C}$. Cells were incubated for $72 \mathrm{~h}$ with the indicated concentrations of the respective drugs and further incubated for $3 \mathrm{~h}$ with 5 $\mathrm{mg} / \mathrm{ml}$ MTT (Merck). Finally, DMSO (Roth) was added and the optical density was measured at 560nm using an Infinite Pro 200 plate reader (Tecan, Switzerland).

\section{Statistical Analysis}

Results for continuous variables are presented as means \pm SEM. Unless stated otherwise, treatment groups were compared with the one-sided Mann-Whitney-U test, tumor growth dynamics were compared by calculating areas under the curve. Chi-squared tests were used to compare CSC frequencies. Contingency tables were compared using Fisher's exact test. P values $<0.05$ were considered statistically significant. Statistical analyses were performed using GraphPad Prism 5.0 (San Diego, CA).

\section{Data availability}

This study includes no data deposited in external repositories. All data generated and analyzed in this study are included within the article and are available from the corresponding author. 


\section{Acknowledgments}

We are indebted to Andrea Wißmann and Kristina Diepold for excellent technical and experimental support. This report includes data that were generated as part of the doctoral thesis of K.W. at Ulm University. Technical illustrations in this manuscript were used from Servier Medical Art under a creative commons attribution 3.0 unported license.

\section{Funding}

Work in the laboratory of P.C.H. is supported by a Max Eder Fellowship of the German Cancer Aid (111746), a German Cancer Aid Priority Program `Translational Oncology’ 70112505, by a Collaborative Research Centre grant (316249678 - SFB 1279) and a graduate college (GRK2254) of the German Research Foundation, and by a Hector Foundation Cancer Research grant (M65.1). B.S.Jr. is supported by a Rámon y Cajal Merit Award (RYC-2012-12104) from the Ministerio de Economía y Competitividad, Spain and a Coordinated grant (GC16173694BARB) from the Fundación Asociación Española Contra el Cáncer (AECC).

\section{Author contribution}

K.W., F.B., B.S.Jr., and P.C.H. conceived the study and designed the experiments. K.W., T.D., K.T., L.L., L.A.S., F.A., V.U., N.A., M.E., A.L., and B.S.Jr. performed the experiments and analyzed the data. M.V.F., F.B., and T.B. designed and performed telomere length analyses. S.M., and C.G., provided technical support for TERT-knockdown constructs. K.W., E.R.A., P.O.F., T.S., A.K., F.B., B.S.Jr., and P.C.H. contributed to the study design, as well as writing and revising the manuscript. B.S.Jr., and P.C.H. acquired funding for the project. All authors contributed to the review and editing of the manuscript.

\section{Conflict of interest}

The authors declare no conflict of interest. 


\section{The Paper Explained}

\section{Problem:}

Despite intensive research pancreatic ductal adenocarcinoma (PDAC) still has a poor prognosis and the therapeutic options are limited. Cancer stem cells (CSCs) are essential for PDAC propagation, for its metastatic spread and chemoresistance. To date the precise mechanism, which preserves the stemness characteristics of CSCs and regulates the unlimited self-renewal potential of these cells is unclear.

\section{Results:}

We discovered that telomerase activity and telomere elongation are essential for the maintenance of the stemness characteristics in pancreatic CSCs. Stemness in pancreatic CSCs is regulated via a feedback loop between stemness factors (NANOG, OCT3/4, SOX2, KLF4) and telomerase activity. The inhibition of telomerase via small molecule inhibitor treatment or genetic knock-down diminished CSCs in human primary pancreatic cancer cells by inducing DNA damage and apoptosis.

\section{Impact:}

Our study demonstrates that telomerase regulation is critical for stemness maintenance in pancreatic CSCs and examines the effects of telomerase inhibition as a potential treatment option of pancreatic cancer. This significantly promotes our understanding of PDAC tumor biology and CSC regulation and may result in improved treatment for pancreatic cancer patients. 


\section{References:}

Bechter OE, Eisterer W, Dlaska M, Kuhr T, Thaler J (2002) CpG island methylation of the hTERT promoter is associated with lower telomerase activity in B-cell lymphocytic leukemia. Exp Hematol 30 26-33

Beier F, Beier CP, Aschenbrenner I, Hildebrandt GC, Brummendorf TH, Beier D (2011) Identification of CD133()/telomerase(low) progenitor cells in glioblastoma-derived cancer stem cell lines. Cell Mol Neurobiol 31: $337-343$

Brassat U, Balabanov S, Bali D, Dierlamm J, Braig M, Hartmann U, Sirma H, Gunes C, Wege H, Fehse B et al (2011) Functional p53 is required for effective execution of telomerase inhibition in BCR-ABL-positive CML cells. Exp Hematol

39: 66-76

Celeghin A, Giunco S, Freguja R, Zangrossi M, Nalio S, Dolcetti R, Rossi AD (2016) Short-term inhibition of TERT induces telomere length-independent cell cycle arrest and apoptotic response in EBV-immortalized and transformed B cells. Cell death \& disease 7

Chan KK, Zhang J, Chia NY, Chan YS, Sim HS, Tan KS, Oh SK, Ng HH, Choo AB (2009) KLF4 and PBX1 directly regulate NANOG expression in human embryonic stem cells. Stem Cells 27: 2114-2125

Chin L, Artandi SE, Shen Q, Tam A, Lee SL, Gottlieb GJ, Greider CW, DePinho RA (1999) p53 deficiency rescues the adverse effects of telomere loss and cooperates with telomere dysfunction to accelerate carcinogenesis. Cell 97: 527538

Clarke M, Dick J, Dirks P, Eaves C, Jamieson C, Jones D, Visvader J, Weissman I, Wahl G (2006) Cancer Stem Cells-Perspectives on Current Status and Future Directions: AACR Workshop on Cancer Stem Cells. Cancer research 66: 9339-9344

Collado M, Blasco MA, Serrano M (2007) Cellular senescence in cancer and aging. Cell 130: 223-233

Conroy T, Desseigne F, Ychou M, Bouche O, Guimbaud R, Becouarn Y, Adenis A, Raoul JL, Gourgou-Bourgade S, de la Fouchardiere C et al (2011) FOLFIRINOX versus gemcitabine for metastatic pancreatic cancer. N Engl J Med 364: 1817-1825

Damm K, Hemmann U, Garin-Chesa P, Hauel N, Kauffmann I, Priepke H, Niestroj C, Daiber C, Enenkel B, Guilliard $\mathrm{B}$ et al (2001) A highly selective telomerase inhibitor limiting human cancer cell proliferation. EMBO J 20: 6958-6968

DeLange T (2005) Shelterin: the protein complex that shapes and safeguards human telomeres. Genes \& Dev 21002110

El-Daly H, Kull M, Zimmermann S, Pantic M, Waller CF, Martens UM (2005) Selective cytotoxicity and telomere damage in leukemia cells using the telomerase inhibitor BIBR1532. Blood 105: 1742-1749

Gallmeier E, Hermann PC, Mueller MT, Machado JG, Ziesch A, De Toni EN, Palagyi A, Eisen C, Ellwart JW, Rivera J et al (2011) Inhibition of ataxia telangiectasia- and Rad3-related function abrogates the in vitro and in vivo tumorigenicity of human colon cancer cells through depletion of the CD133(+) tumor-initiating cell fraction. Stem Cells 29: 418-429

Griffith LG, Swartz MA (2006) Capturing complex 3D tissue physiology in vitro. Nature reviews Molecular cell biology 7: 211-224

Guilleret I, Yan P, Grange F, Braunschweig R, Bosman FT, Benhattar J (2002) Hypermethylation of the human telomerase catalytic subunit (hTERT) gene correlates with telomerase activity. Int J Cancer 101: 335-341 
Hermann PC, Huber SL, Herrler T, Aicher A, Ellwart JW, Guba M, Bruns CJ, Heeschen C (2007) Distinct populations of cancer stem cells determine tumor growth and metastatic activity in human pancreatic cancer. Cell stem cell 1: 313323

Hoffmeyer K, Raggioli A, Rudloff S, Anton R, Hierholzer A, Del Valle I, Hein K, Vogt R, Kemler R (2012) Wnt/betacatenin signaling regulates telomerase in stem cells and cancer cells. Science 336: 1549-1554

Hotta A, Cheung AY, Farra N, Vijayaragavan K, Seguin CA, Draper JS, Pasceri P, Maksakova IA, Mager DL, Rossant J et al (2009) Isolation of human iPS cells using EOS lentiviral vectors to select for pluripotency. Nat Methods 6: 370376

Hsieh MH, Chen YT, Lee YH, Lu J, Chien CL, Chen HF, Ho HN, Yu CJ, Wang ZQ, Teng SC (2017) PARP1 controls KLF4-mediated telomerase expression in stem cells and cancer cells. Nucleic Acids Res 45: 10492-10503

Hu Y, Smyth GK (2009) ELDA: extreme limiting dilution analysis for comparing depleted and enriched populations in stem cell and other assays. J Immunol Methods 347: 70-78

Hummel S, Ventura Ferreira MS, Heudobler D, Huber E, Fahrenkamp D, Gremse F, Schmid K, Muller-Newen G, Ziegler P, Jost E et al (2015) Telomere shortening in enterocytes of patients with uncontrolled acute intestinal graftversus-host disease. Blood 126: 2518-2521

Joseph I, Tressler R, Bassett E, Harley C, Buseman CM, Pattamatta P, Wright WE, Shay JW, Go NF (2010) The telomerase inhibitor imetelstat depletes cancer stem cells in breast and pancreatic cancer cell lines. Cancer research 70 : 9494-9504

Kim W, Ludlow AT, Min J, Robin JD, Stadler G, Mender I, Lai TP, Zhang N, Wright WE, Shay JW (2016) Regulation of the Human Telomerase Gene TERT by Telomere Position Effect-Over Long Distances (TPE-OLD): Implications for Aging and Cancer. PLoS biology 14: e2000016

Lerma L, Alcala S, Piñero C, Torres M, Martin B, Lim F, Sainz B, Tabarés E (2016) Expression of the immediate early IE180 protein under the control of the hTERT and CEA tumor-specific promoters in recombinant pseudorabies viruses: Effects of IE180 protein on promoter activity and apoptosis induction. . Virology 488: 9-19

Li C, Heidt DG, Dalerba P, Burant CF, Zhang L, Adsay V, Wicha M, Clarke MF, Simeone DM (2007) Identification of pancreatic cancer stem cells. Cancer research 67: 1030-1037

Liu CC, Ma DL, Yan TD, Fan X, Poon Z, Poon LF, Goh SA, Rozen SG, Hwang WY, Tergaonkar V et al (2016) Distinct Responses of Stem Cells to Telomere Uncapping-A Potential Strategy to Improve the Safety of Cell Therapy. Stem Cells 34: 2471-2484

Liu L (2017) Linking Telomere Regulation to Stem Cell Pluripotency. Trends Genet 33: 16-33

Lonardo E, Hermann PC, Mueller M-T, Huber S, Balic A, Miranda-Lorenzo I, Zagorac S, Alcala S, RodriguezArabaolaza I, Ramirez JC et al (2011) Nodal/Activin signaling drives self-renewal and tumorigenicity of pancreatic cancer stem cells and provides a target for combined drug therapy. Cell stem cell

Marian CO, Wright WE, Shay JW (2010) The effects of telomerase inhibition on prostate tumor-initiating cells. Int J Cancer 127: 321-331

Mueller MT, Hermann PC, Witthauer J, Rubio-Viqueira B, Leicht SF, Huber S, Ellwart JW, Mustafa M, Bartenstein P, D'Haese JG et al (2009) Combined targeted treatment to eliminate tumorigenic cancer stem cells in human pancreatic cancer. Gastroenterology 137: 1102-1113 
bioRxiv preprint doi: https://doi.org/10.1101/2020.11.02.361931; this version posted December 23, 2020. The copyright holder for this preprint (which was not certified by peer review) is the author/funder. All rights reserved. No reuse allowed without permission.

Nakatake Y, Fukui N, Iwamatsu Y, Masui S, Takahashi K, Yagi R, Yagi K, Miyazaki J, Matoba R, Ko MS et al (2006) Klf4 cooperates with Oct3/4 and Sox 2 to activate the Lefty1 core promoter in embryonic stem cells. Mol Cell Biol 26: $7772-7782$

Rahib L, Smith BD, Aizenberg R, Rosenzweig AB, Fleshman JM, Matrisian LM (2014) Projecting cancer incidence and deaths to 2030: the unexpected burden of thyroid, liver, and pancreas cancers in the United States. Cancer research 74: 2913-2921

Sexton AN, Regalado SG, Lai CS, Cost GJ, O'Neil CM, Urnov FD, Gregory PD, Jaenisch R, Collins K, Hockemeyer D (2014) Genetic and molecular identification of three human TPP1 functions in telomerase action: recruitment, activation, and homeostasis set point regulation. Genes Dev 28: 1885-1899

Shay JW, Wright WE (2019) Telomeres and telomerase: three decades of progress. Nature reviews Genetics 20: 299309

Siegel RL, Miller KD, Jemal A (2015) Cancer statistics, 2015. CA: a cancer journal for clinicians 65: 5-29

Takai A., Nakano M., Saito K., Haruno R., Watanabe T. M., Ohyanagi T. , Jin T., Okada Y., Nagai T. (2015) Expanded palette of Nano-lanterns for real-time multicolor luminescence imaging. Proc Natl Acad Sci U S A 112 : 4352-4356

Takai H, Smogorzewska A, de Lange T (2003) DNA Damage Foci at Dysfunctional Telomeres. Current Biology 13: 1549-1556

Vinagre J, Almeida A, Populo H, Batista R, Lyra J, Pinto V, Coelho R, Celestino R, Prazeres H, Lima L et al (2013) Frequency of TERT promoter mutations in human cancers. Nature communications 4: 2185

Von Hoff DD, Ervin T, Arena FP, Chiorean EG, Infante J, Moore M, Seay T, Tjulandin SA, Ma WW, Saleh MN et al (2013) Increased survival in pancreatic cancer with nab-paclitaxel plus gemcitabine. N Engl J Med 369: 1691-1703

Wong CW, Hou PS, Tseng SF, Chien CL, Wu KJ, Chen HF, Ho HN, Kyo S, Teng SC (2010) Kruppel-like transcription factor 4 contributes to maintenance of telomerase activity in stem cells. Stem Cells 28: 1510-1517

Wright WE, Piatyszek MA, Rainey WE, Byrd W, Shay JW (1996) Telomerase activity in human germline and embryonic tissues and cells. Dev Genet 18: 173-179

Yang C, Przyborski S, Cooke MJ, Zhang X, Stewart R, Anyfantis G, Atkinson SP, Saretzki G, Armstrong L, Lako M (2008) A key role for telomerase reverse transcriptase unit in modulating human embryonic stem cell proliferation, cell cycle dynamics, and in vitro differentiation. Stem Cells 26: 850-863

Zhang HH, Li SZ, Zhang ZY, Hu XM, Hou PN, Gao L, Du RL, Zhang XD (2014) Nemo-like kinase is critical for p53 stabilization and function in response to DNA damage. Cell death and differentiation 21: 1656-1663 


\section{Figure legends:}

\section{Figure 1 - Telomerase activity stabilizes telomere length in pancreatic CSCs.}

A Telomere length in primary PDAC cells (red dot) with respect to patient age measured by FlowFISH.

B Schematic illustration showing the CSC enrichment methods for CD133 and Aldefluor via FACS or in sphere culture.

C, D, E RT-qPCR analysis of TERT and TERF1 mRNA levels in primary pancreatic cancer stem cells enriched and selected by FACS for CD133 (C), or Aldefluor (D), and cultured as spheres (E) (CSCs) vs corresponding control (non CSCs) ( $\mathrm{n}=$ at least 3 independent experiments).

F Immunofluorescence staining and quantification for TERT (red) in CD133- and CD133+ FACSorted cells ( $\mathrm{n}=5$ independent experiments). Cells were counterstained with DAPI (nuclear marker, blue).

G, H, I Telomerase activity measurement in CD133 (G) or Aldefluor $(\mathrm{H})$ negative vs positive cells and in adherent vs sphere cell-cultures (I) ( $\mathrm{n}=3$ independent FACSortings and sphere culture experiments).

J Representative pictures of Q-FISH with telomeres (green) and DAPI (blue) and violin plot showing telomere length analysis in CD133 negative (non CSCs) and positive cells (CSCs). The mean is depicted in numbers and as black line. $\mathrm{n} \geq 3$ from independent FACSortings with each $>150$ measurements per group.

Data information: In (C-J), data are presented as mean $\pm \mathrm{SEM} .{ }^{*} \mathrm{P} \leq 0.05$ (Mann-Whitney-U test). CSC, cancer stem cells; FACS, fluorescence activated cell sorting; FlowFISH, Flow fluorescence insitu hybridization; PDAC, pancreatic ductal adenocarcinoma; Q-FISH, quantitative fluorescent in situ hybridization; RT-qPCR, reverse transcriptase polymerase chain reaction.

\section{Figure 2 Interplay between pluripotency / stemness factors and telomerase activity in} pancreatic CSCs.

A, B RT-qPCR analysis of pluripotency / stemness-associated genes in primary pancreatic CSCs enriched by CD133 expression (A) or Aldefluor activity (B) vs CD133- and Aldefluor- non-CSCs (n $=$ at least 3 independent FACSortings).

C, D, E, F Quantitative RT-PCR (C), telomerase activity (D), flow cytometry analysis for CD133 (E) and sphere formation capability (F), compared in NANOG negative cells (white) vs NANOG positive cells (yellow) using a NANOG-YNL reporter.

G Schematic illustration and quantification on the production of virions in parental pseudorabies viruses (PRV-NIA3 and vBecker) compared to telomerase activity-dependent virus production (PRV-TER) in adherent HPDE and Panc185 cells as compared to Panc185 spheres. 
$\mathrm{H}$ TERT promoter mutation analysis of C250T and C228T sites in 6 primary pancreatic cancer cells (Panc215, Panc354, Panc185, Panc286, Panc253, and Panc010414).

I Gene expression levels of stemness / pluripotency genes and TERT in HEK293T cells after inducing expression of SOX2, OCT3/4, KLF4 and NANOG, compared to the empty vector control $(\mathrm{n}=4$ independent experiments).

Data information: In (A - G and I), data are represented as mean \pm SEM. $* \mathrm{P} \leq 0.05$ (Mann-WhitneyU test).

YNL, yellow Nano-latern reporter system.

\section{Figure 3 Targeting telomerase activity with a small molecule inhibitor (BIBR1532)}

A, B The effects of BIBR1532 treatment on telomerase activity (A), and telomere length (B) in CD133- cells (blue) and in the CD133+ CSC population (red) were quantified. For illustrative purposes, the telomere length measurements of Fig1H were re-used here (indicated by transparent color). The mean is depicted in numbers and as black line, >150 measurements per group.

C Quantification of gH2AX foci (>50 cells per group) in CD133- and CD133+ cells after 7 days vehicle or BIBR1532 treatment. Quantification and representative pictures of immunofluorescence staining of $\mathrm{gH} 2 \mathrm{AX}$ foci are provided.

D Senescent cells quantified by flow cytometry staining for SA-beta-Gal in CD133- (non CSCs) and CD133+ (CSCs) after BIBR1532 or solvent treatment (7 days).

E, F Apoptosis quantified by flow cytometry using double staining for CD133 and AnnexinV or (E) Caspase3/7 (F).

Data information: In (A-F), data are represented as mean \pm SEM. $\mathrm{n}=3$ independent experiments. *P $\leq 0.05$ (Mann-Whitney-U test).

\section{Figure 4 Telomerase inhibition as treatment strategy for pancreatic cancer (stem) cells}

A Effects of the small molecule telomerase inhibitor BIBR1532 on the expression of TERT and pluripotency-associated genes as measured by RT-qPCR ( $\mathrm{n}=4$ independent experiments).

B Flow cytometry analyses for CD133 or Aldefluor after BIBR1532 or solvent treatment ( $\mathrm{n}=3$ independent experiments).

C Quantification and representative pictures of spheres after BIBR1532 treatment $(\mathrm{n}=3$ independent experiments).

D Schematic of BIBR1532 pre-treatment and in vivo experiment, number of tumorigenic cells within the whole population shown as cancer stem cell (CSC) frequencies as determined by extreme limiting dilution assays (ELDA) in nude mice, and tumor volume measured after injection of 100.000 BIBR1532 or solvent treated Panc215 and Panc354 cells ( $n \geq 4$ mice for each group). 
E Viability of PDX-derived organoids treated with gemcitabine and BIBR1532 at the indicated concentrations.

F Quantification and representative pictures of sphere formation assays after single agent or combination treatment with gemcitabine, Olaparib, and BIBR1532 ( $\mathrm{n}=5$ independent experiments). Data information: In (A-C; E-F) data are represented as mean \pm SEM. ${ }^{*} \mathrm{P} \leq 0.05$ (Mann-Whitney-U test). In (G) data are represented as mean \pm SEM. $* \mathrm{P} \leq 0.05$ (Area under the curve).

\section{Figure 5 Knockdown of TERT diminishes pancreatic cancer stem cells}

A, B Gene expression of TERT (A) and telomerase activity (B) in Panc215 and Panc354 cells transduced with shRNA-340159 and shRNA-340160 compared to scrambled (shSCR) control. TERT mediated knockdown in Panc215 and Panc354 cells.

D, E Flow cytometry analyses for CD133 (D) and sphere formation (E) upon TERT knock-down (KD) compared to scrambled (shSCR) control.

F Quantification of apoptosis in shSCR and shTERT transduced cells (Panc215 and Panc354) using flow cytometry analysis for AnnexinV ( $\mathrm{n}=$ at least 3 independent experiments).

$\mathrm{G}$ In vivo tumor-initiating potential with CSC frequencies (number of tumorigenic cells within the whole population) as determined by ELDA in nude mice after TERT-KD compared to scrambled control ( $\mathrm{n}=8$ animals).

$\mathrm{H}$ Schematic illustration of in vivo experiment with cells carrying an inducible TERT-KD (Dox days before s.c. xenografting in nude mice. Graph shows time-dependent growth of subcutaneously engrafted tumors ( $n=4$ mice per group).

I Schematic illustration of in vivo experiment and visual representation of time-dependent tumor growth of subcutaneously (s.c.) engrafted tumors arising from TERT-KD and SCR cells, over the course of doxycycline treatment 28 days after s.c. xenografting in nude mice ( $\mathrm{n}=6$ mice per group). Tumor growth is depicted until the first control mice had to be sacrificed.

J Gene expression of TERT and telomerase activity compared in TERT-KD and SCR tumors (induced with DOX at day 28) after sacrificing mice 43 days after xenografting.

$\mathrm{K}$ representative $\mathrm{H} \& \mathrm{E}$ staining of at day 43 explanted TERT-KD and scrambled tumors (induced with DOX at day 28).

Data information: In (A-G and $\mathrm{J}$ ) data are represented as mean \pm SEM. ${ }^{*} \mathrm{P} \leq 0.05$ (Mann-Whitney-U test). In ( $\mathrm{H}$ and $\mathrm{I})$ data are represented as mean \pm SEM. $* \mathrm{P} \leq 0.05$ (Area under the curve).

AUC, area under the curve; ELDA, extreme limiting dilution assay; DOX, doxycycline; KD, knockdown; s.c.; subcutaneous; shSCR, scrambled control; shTERT, shRNA-340160. 


\section{Supplementary Figure 1 Additional Data}

745 A Representative pictures and Q-FISH telomere length analysis in Aldefluor positive and negative 746 cells. The mean is depicted in numbers and as black line, $>150$ measurements per group. B TERT promoter mutation analysis of C250T and C228T sites in five established pancreatic cancer cell lines.

749 C MTT assay to determine the respective $\mathrm{IC}_{50}$ of BIBR1532 in the utilized primary pancreatic cancer 750 cells. vehicle (Ctrl) or BIBR1532 treatment.

753 F Representative pictures of explanted subcutaneous tumors from Panc354 ELDA assays. 


\section{Supplementary file 1}

\section{Table 1 Antibodies}

\begin{tabular}{lcc}
\hline & Application & Manufacturer \\
\hline$\alpha$-hu-CD133/1-(AC133)-APC & FC & MiltenyiBiotec (Cat no. 130090826) \\
\hline$\alpha$-hu-CD133/1-(AC133)-PE & FC & MiltenyiBiotec (Cat no. 130080801) \\
\hline$\alpha$-hu-CD133/1-(AC133)-PE-VIO770 & FC & MiltenyiBiotec (Cat no. 130102358) \\
\hline$\alpha$-hu-EpCAM-APC & FC & BD Biosciences (Cat no. 347200) \\
\hline$\alpha-\gamma$-H2A.X (Ser139) & IF & Merck (Cat no. 05-636)
\end{tabular}

758

Table 2 RT qPCR Primers

\begin{tabular}{|c|c|c|}
\hline & Antisense & Manufacturer \\
\hline \multirow[t]{2}{*}{ HPRT } & & Qiagen (Cat no. \\
\hline & & QT000590669) \\
\hline NANOG & & Qiagen (Cat no. QT01025850) \\
\hline LGR5 & & Qiagen (Cat no. QT00027720) \\
\hline \multirow[t]{2}{*}{ TERT } & & Qiagen (Cat no. \\
\hline & & QT000734099) \\
\hline TRF1 & & Qiagen (Cat no. QT00059528) \\
\hline OCT3/4 & TGAACCTCAGCTACAAACAGGTG AACTGCATGCAGGACTGCAGAG & ThermoFisher \\
\hline KLF4 & ACCCACACAGGTGAGAAACC & ThermoFisher \\
\hline SOX2 & AGAACCCCAAGATGCACAAC & ThermoFisher \\
\hline ACX & GCGCGGCTTA(CCCTTA $)_{3}$ CCCTAA & SigmaAldrich \\
\hline TS & AATCCGTCGAGCAGAGTT & SigmaAldrich \\
\hline
\end{tabular}




\section{Figure 1}

A

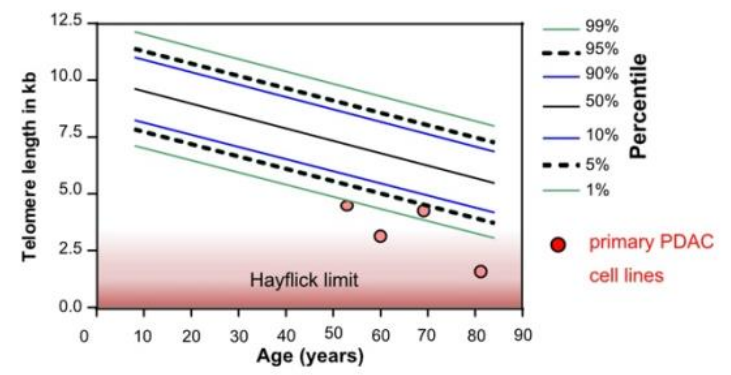

D

E

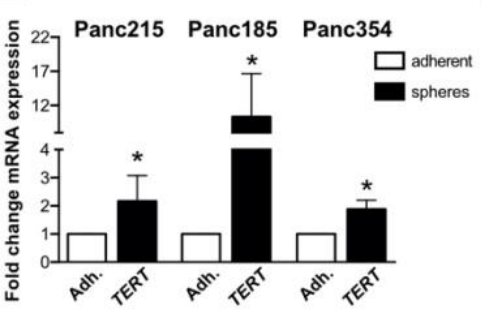

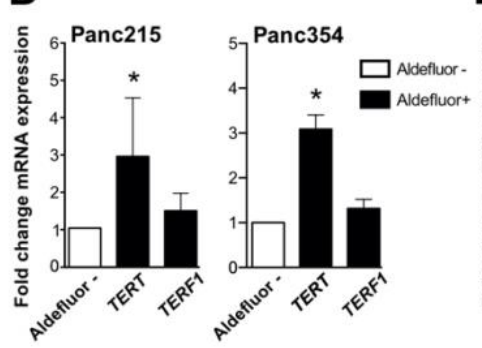

C

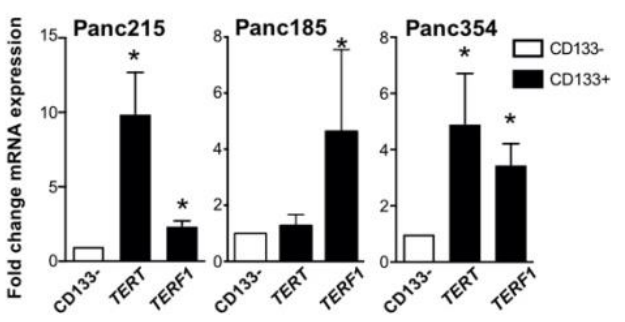

$\mathbf{F}$
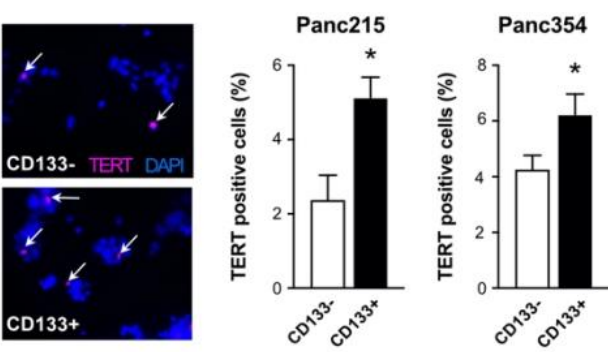

G

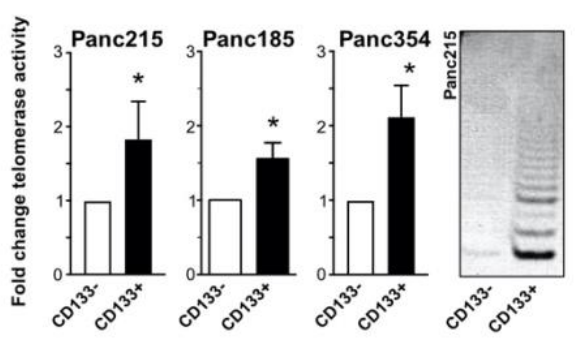

H
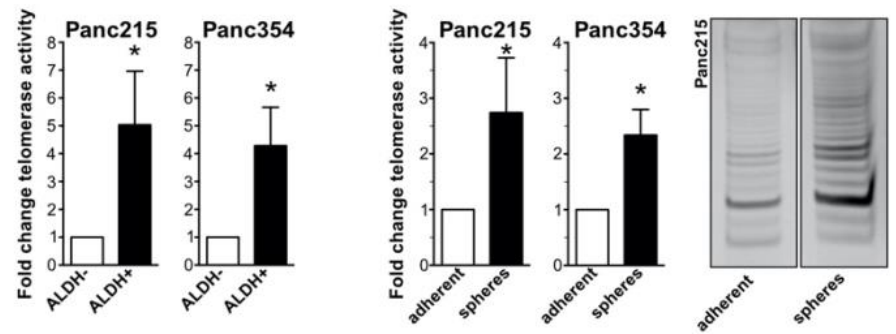

J
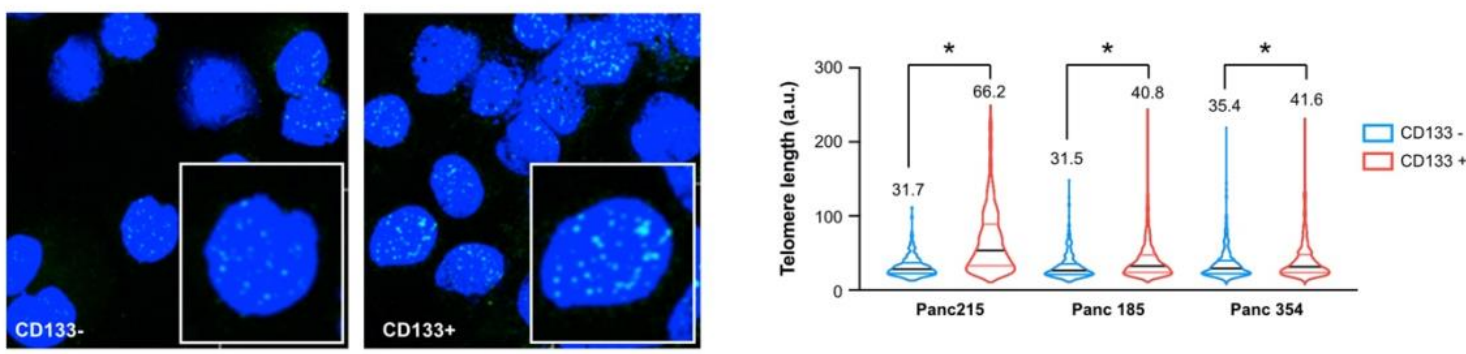


\section{Figure 2}
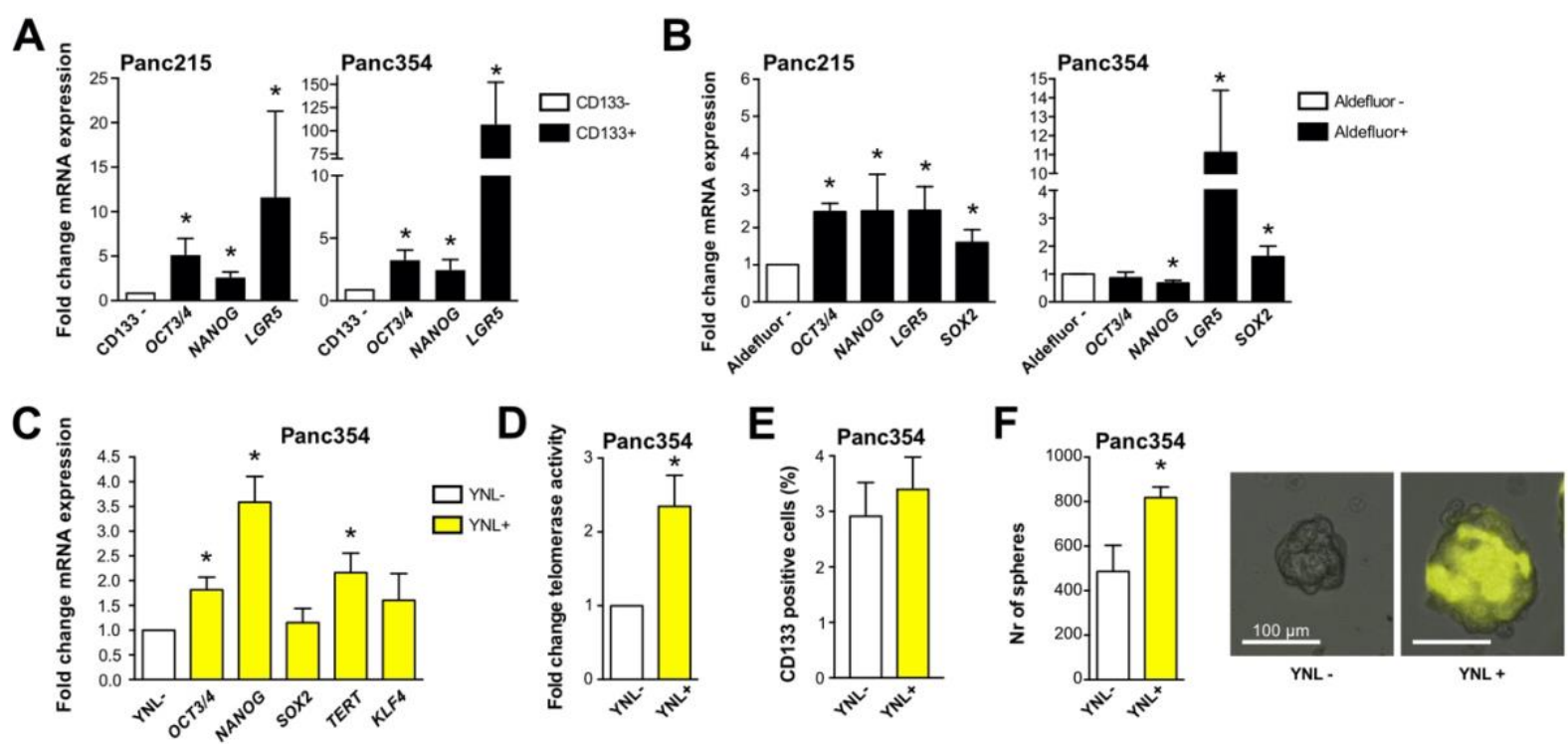

G
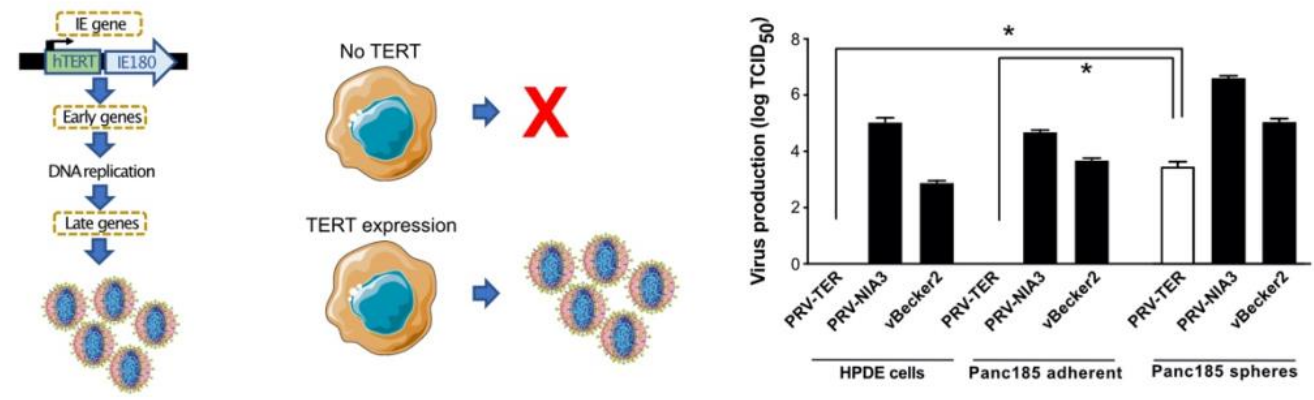

H
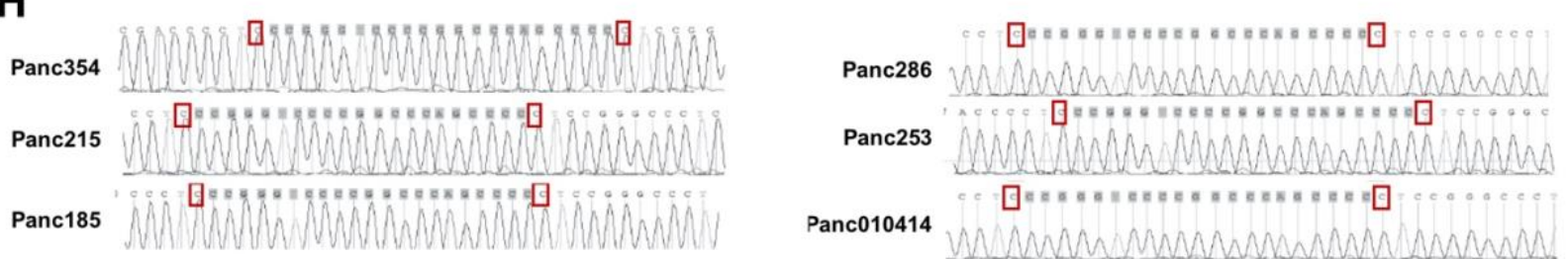

I
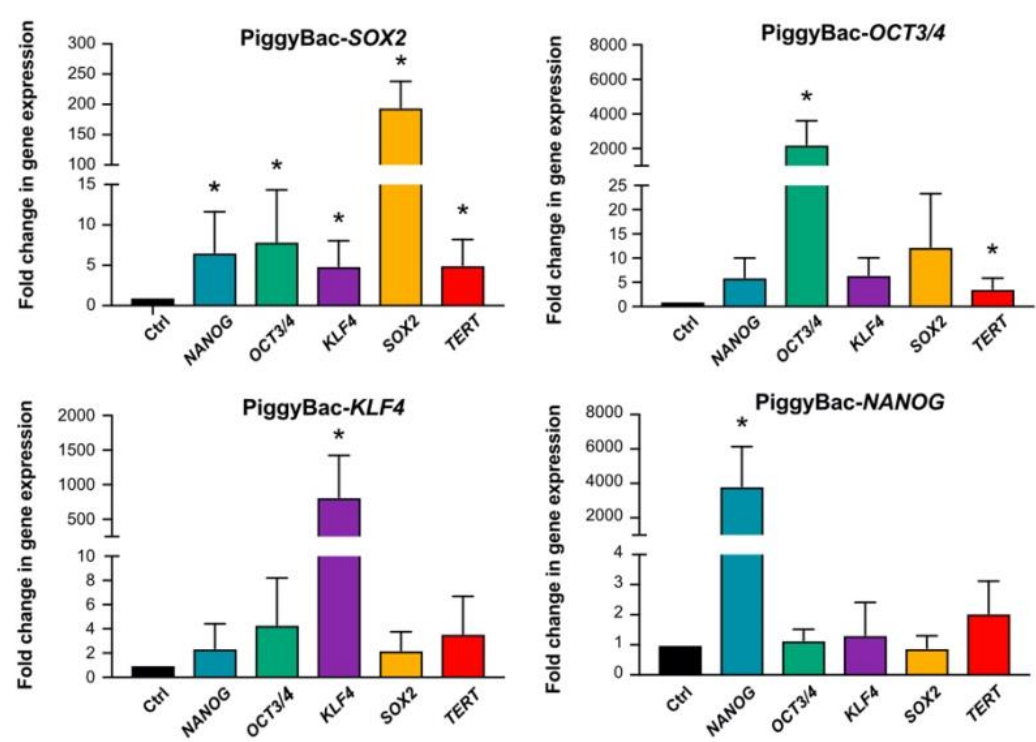


\section{Figure 3}

A

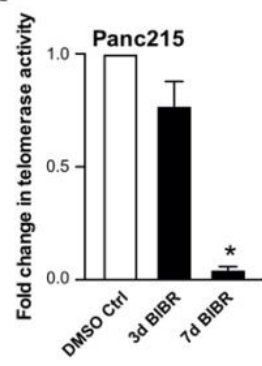

C
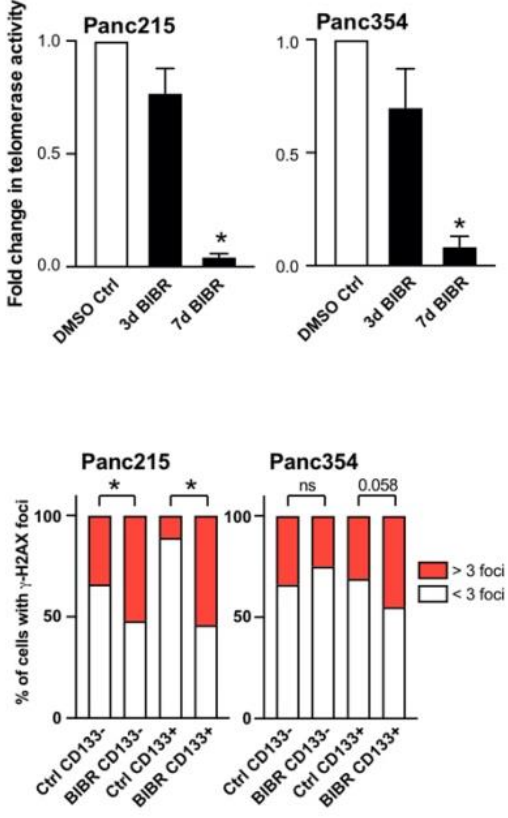

B
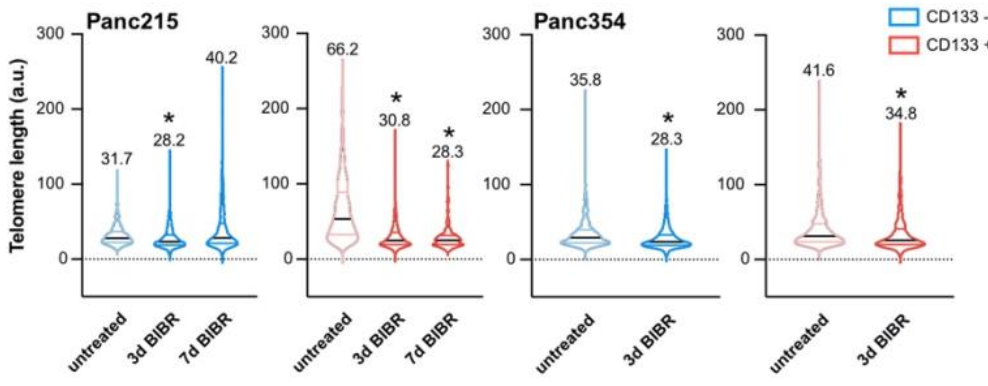

D

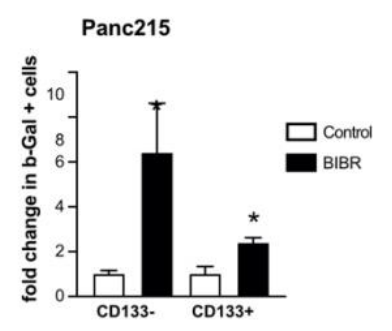

E
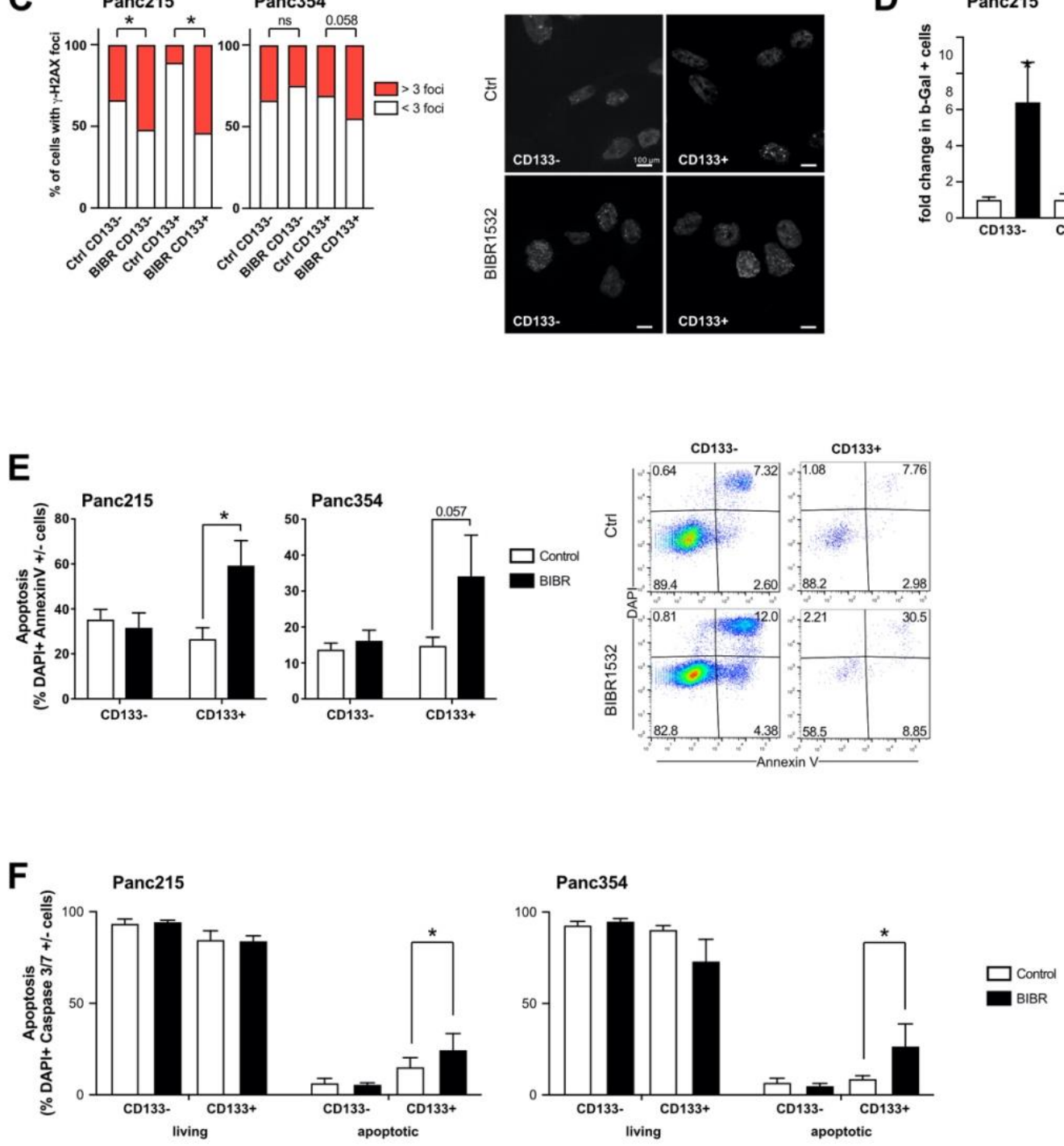


\section{Figure 4}

A
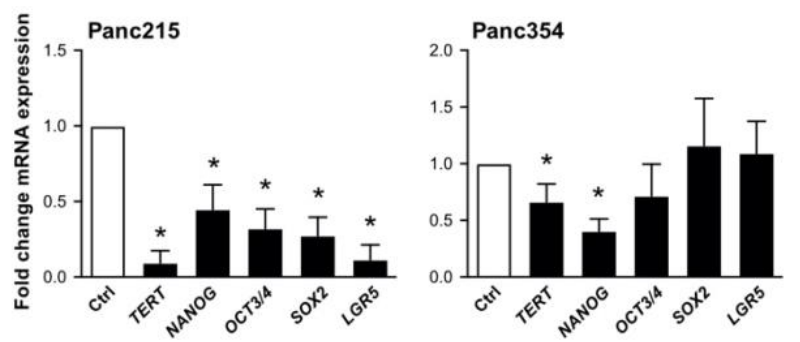

B

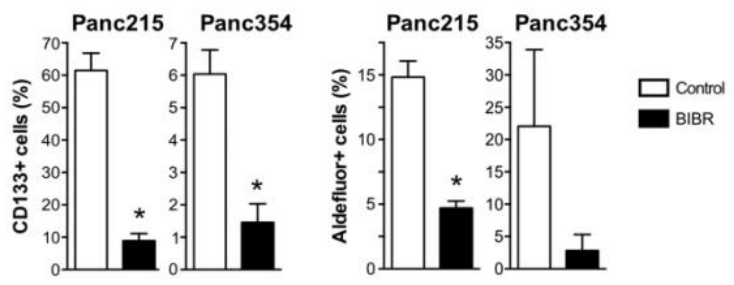

C
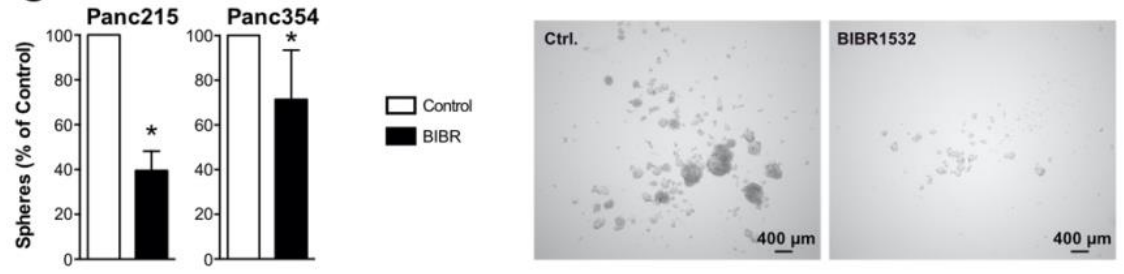

D

Panc215 CSC Frequency in nude mice:
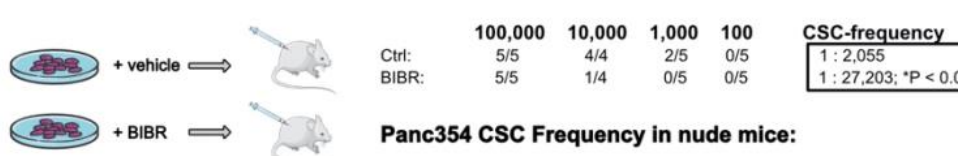

\begin{tabular}{lllllll} 
& Ctr: & $5 / 5$ & $4 / 4$ & $2 / 5$ & $0 / 5$ & $1: 2,055$ \\
BIBR: & $5 / 5$ & $1 / 4$ & $0 / 5$ & $0 / 5$ & $1: 27,203 ; \cdot P<0.05$ \\
\hline & & & & &
\end{tabular}

$400 \mu \mathrm{m}$

Panc354 CSC Frequency in nude mice:
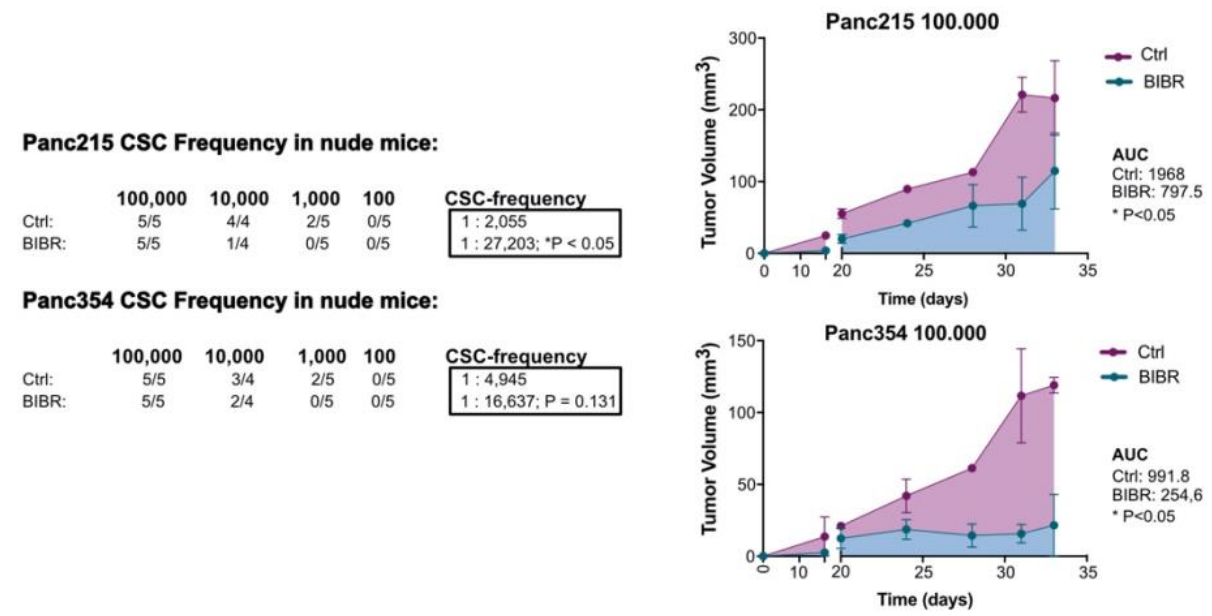

$\mathbf{E}$
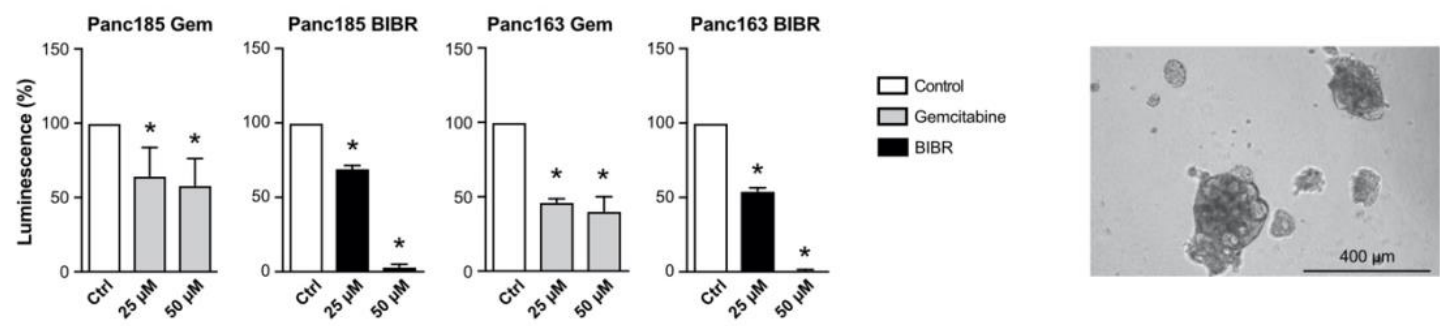

F
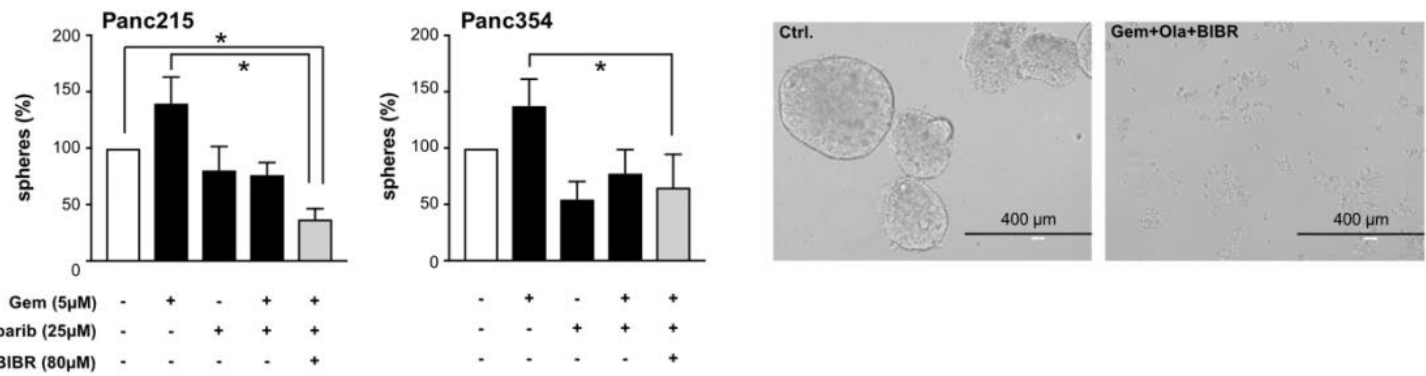


\section{Figure 5}

A
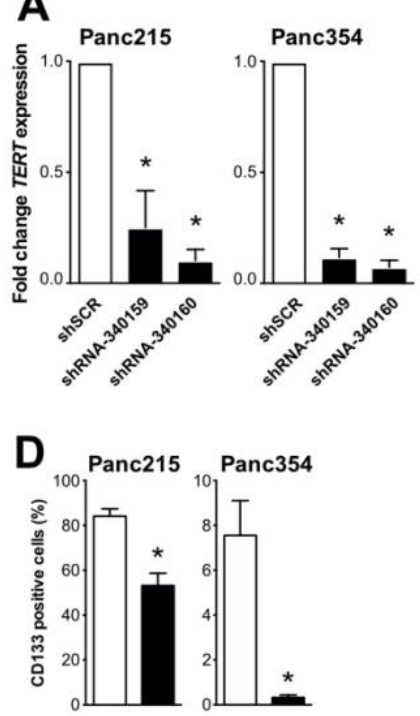

B
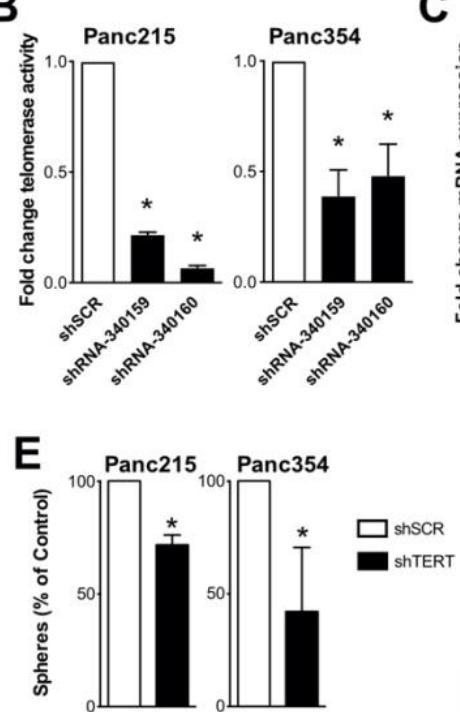

C
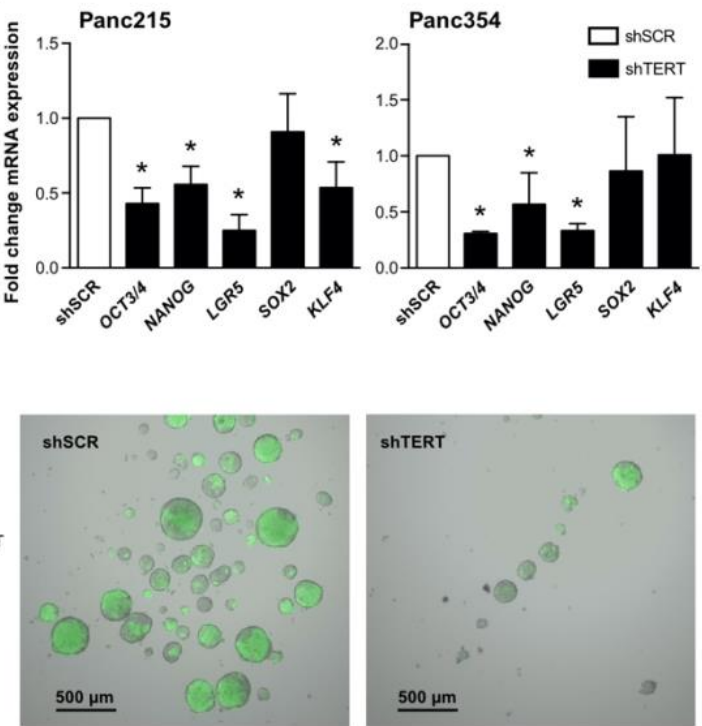

G

Panc215 CSC Frequency in nude mice:

$\begin{array}{lccccc} & 100,000 & 10,000 & 1,000 & 100 & \text { CSC-frequency } \\ \text { shSCR: } & 8 / 8 & 8 / 8 & 6 / 8 & 5 / 8 & 1: 400 \\ \text { shTERT: } & 6 / 8 & 7 / 8 & 5 / 8 & 4 / 8 & 1: 15,400 ; * P<0.05\end{array}$

Panc354 CSC Frequency in nude mice:

$\begin{array}{lccccc} & 100,000 & 10,000 & 1,000 & 100 & \text { CSC-frequency } \\ \text { shSCR: } & 8 / 8 & 8 / 8 & 6 / 8 & 4 / 8 & 1: 458 \\ \text { shTERT: } & 5 / 8 & 4 / 8 & 1 / 8 & 3 / 8 & 1: 40,236 ; * P<0.05\end{array}$
H

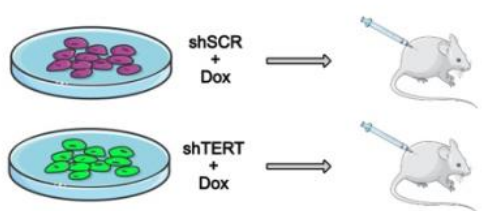

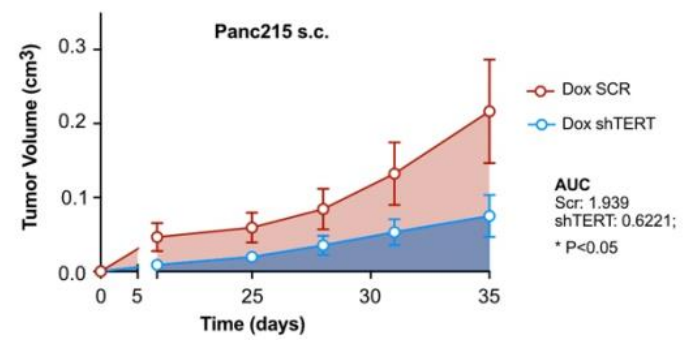

I

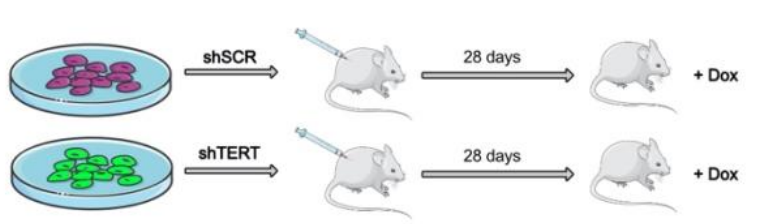

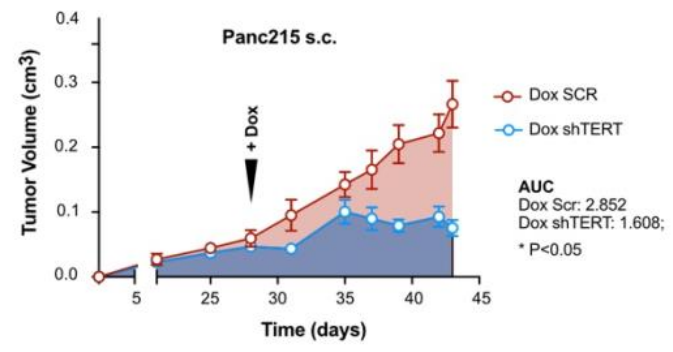

J

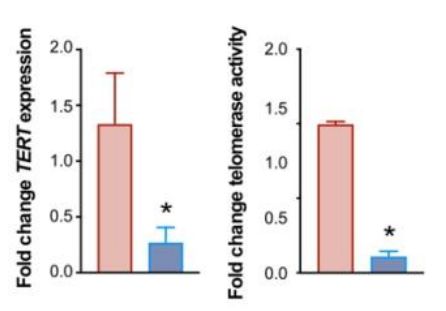

K

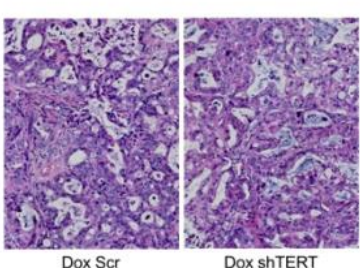




\section{Suppl. Figure1}

\section{A}
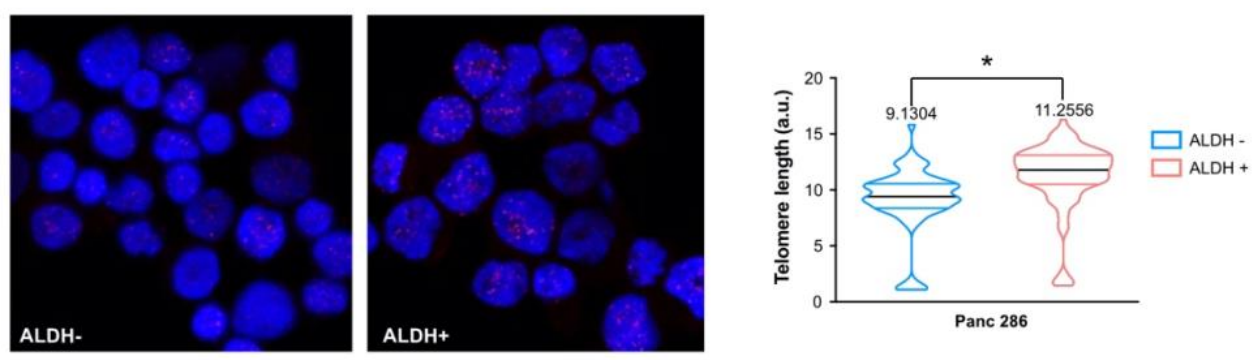

B
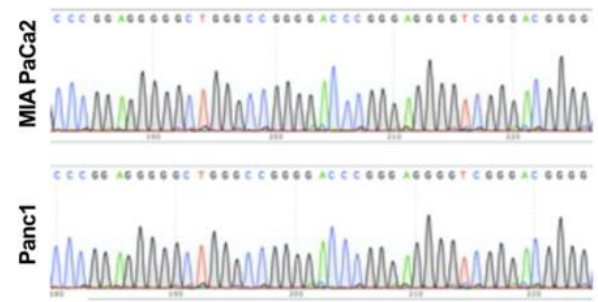

怘
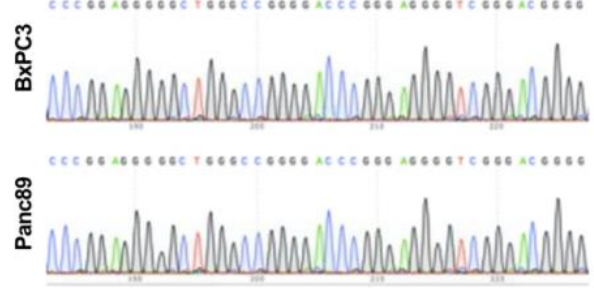

C

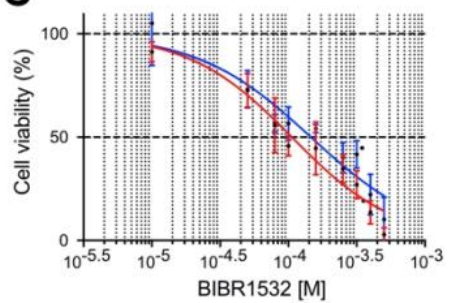

- Panc215 IC 50: $146.2 \mu \mathrm{M}$

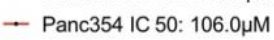

D

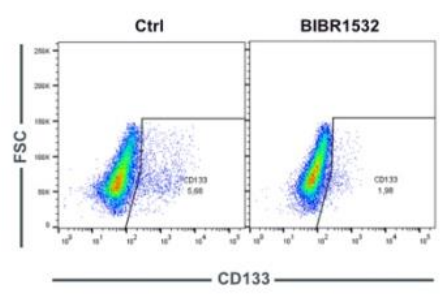

$\mathbf{E}$

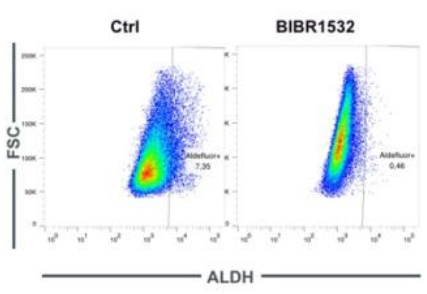

$\mathbf{F}$

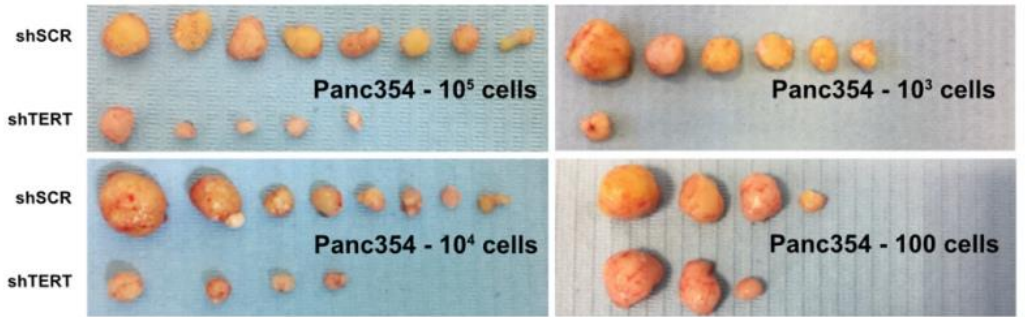

